\title{
The Wnt Effector TCF712 Promotes Oligodendroglial Differentiation by Repressing Autocrine BMP4-Mediated Signaling
}

\author{
${ }^{\text {D }}$ Sheng Zhang, ${ }^{1,2 *}$ Yan Wang, ${ }^{1,2 *}$ Xiaoqing Zhu, ${ }^{1,2}$ Lanying Song, ${ }^{1,2}$ Xinhua Zhan, ${ }^{1}$ Edric Ma, ${ }^{1,2}$ \\ Jennifer McDonough, ${ }^{3}$ Hui Fu, ${ }^{4}$ Franca Cambi, ${ }^{5}{ }^{1}$ Judith Grinspan, ${ }^{6}$ and ${ }^{\circledR}$ Fuzheng Guo ${ }^{1,2}$ \\ ${ }^{1}$ Department of Neurology, School of Medicine, University of California, Davis, California, 95817, ${ }^{2}$ Institute for Pediatric Regenerative Medicine \\ (IPRM), Shriners Hospitals for Children/UC Davis School of Medicine, Sacramento, California, 95817, ${ }^{3}$ Department of Biological Sciences, Kent \\ State University, Kent, Ohio, 44242, ${ }^{4}$ Division of Life Sciences and Medicine, School of Basic Medical Sciences, University of Science and \\ Technology of China, Anhui, China, 230027, ${ }^{5}$ Department of Neurology, Pittsburgh University, Pittsburgh, Pennsylvania, 15260, and ${ }^{6}$ Department \\ of Neurology, The Children's Hospital of Philadelphia, Philadelphia, Pennsylvania, 19104
}

Promoting oligodendrocyte (OL) differentiation represents a promising option for remyelination therapy for treating the demyelinating disease multiple sclerosis (MS). The Wnt effector transcription factor 7-like 2 (TCF712) was upregulated in MS lesions and had been proposed to inhibit OL differentiation. Recent data suggest the opposite yet underlying mechanisms remain elusive. Here, we unravel a previously unappreciated function of TCF712 in controlling autocrine bone morphogenetic protein (BMP)4-mediated signaling. Disrupting TCF712 in mice of both sexes results in oligodendroglial-specific BMP4 upregulation and canonical BMP4 signaling activation in vivo. Mechanistically, TCF7l2 binds to Bmp4 gene regulatory element and directly represses its transcriptional activity. Functionally, enforced TCF712 expression promotes OL differentiation by reducing autocrine BMP4 secretion and dampening BMP4 signaling. Importantly, compound genetic disruption demonstrates that oligodendroglial-specific BMP4 deletion rescues arrested OL differentiation elicited by TCF712 disruption in vivo. Collectively, our study reveals a novel connection between TCF712 and BMP4 in oligodendroglial lineage and provides new insights into augmenting TCF712 for promoting remyelination in demyelinating disorders such as MS.

Key words: BMP4 repression; canonical Wnt//-catenin; myelination; oligodendrocyte differentiation; TCF7l2/TCF4; Wnt effector

Significance Statement

Incomplete or failed myelin repairs, primarily resulting from the arrested differentiation of myelin-forming oligodendrocytes (OLs) from oligodendroglial progenitor cells, is one of the major reasons for neurologic progression in people affected by multiple sclerosis (MS). Using in vitro culture systems and in vivo animal models, this study unraveled a previously unrecognized autocrine regulation of bone morphogenetic protein (BMP)4-mediated signaling by the Wnt effector transcription factor 7-like 2 (TCF712). We showed for the first time that TCF712 promotes oligodendroglial differentiation by repressing BMP4mediated activity, which is dysregulated in MS lesions. Our study suggests that elevating TCF712 expression may be possible in overcoming arrested oligodendroglial differentiation as observed in MS patients.

Received Sep. 12, 2020; revised Dec. 2, 2020; accepted Jan. 1, 2021

*S.Z. and Y.W. are co-first authors.

Author contributions: J.G. and F.G. designed research; S.Z., Y.W., X.Zhu, L.S., and E.M. performed research; X.Zha., E.M., H.F., J.M., F.C., J.G., and F.G. contributed unpublished reagents/analytic tools; S.Z., Y.W., X.Zhu, L.S., H.F., F.C., J.G., and F.G. analyzed data; S.Z., Y.W., J.G., and F.G. wrote the paper.

This work was funded by National Institutes of Health/National Institute of Neurological Disorders and Stroke Grants R21NS109790 and R01NS094559 and by Shriners Hospitals for Children Grants 85107-NCA-19, 84553-NCA-18, and 84307-NCAL. We thank Dr. Andreas Hecht (University of Freiburg, Germany) for providing TCF7I2-E2, M1, and S2 expression vectors and Dr. Gino Cortopassi (University of California, Davis) for providing U87 cells.

The authors declare no competing financial interests.

Correspondence should be addressed to Fuzheng Guo at fzguo@ucdavis.edu.

https://doi.org/10.1523/JNEUROSCI.2386-20.2021

Copyright $\odot 2021$ the authors

\section{Introduction}

Blocked differentiation of myelin-forming oligodendrocytes (OLs) from oligodendrocyte precursor (or progenitor) cells (OPCs) is a major cause for remyelination failure in multiple sclerosis (MS), the most common demyelinating disorder (Franklin and Gallo, 2014). Promoting OL differentiation represents a promising option for remyelination therapy in combination with current immunomodulatory therapy for treating MS (Harlow et al., 2015). Identifying upregulated molecular targets in MS will provide new insights into designing remyelination therapeutics. The upregulation of the Wnt effector transcription 
factor 7-like 2 (TCF712) in MS brain lesions (Fancy et al., 2009) suggest that it might be a potential target for overcoming OL differentiation blockade (Fu et al., 2009).

TCF712 is one of the four members (TCF1, TCF711, TCF712, and LEF1) of the TCF/LEF1 family that transcriptionally activate the canonical Wnt/ $\beta$-catenin signaling (Cadigan, 2012). Based on the inhibitory role of $\mathrm{Wnt} / \beta$-catenin activation in OL differentiation (Guo et al., 2015), TCF7l2 had been previously proposed as a negative regulator of OL differentiation by transcriptionally activating $\mathrm{Wnt} / \beta$-catenin signaling ( $\mathrm{He}$ et al., 2007; Fancy et al., 2009). However, subsequent genetic studies have convincingly demonstrated that TCF712 is a positive regulator of OL differentiation that TCF712 play a minor role in activating $\mathrm{Wnt} / \beta$-catenin in oligodendroglial lineage cells (Fu et al., 2009; Hammond et al., 2015; Zhao et al., 2016). In this context, the molecular mechanisms underlying TCF712-regulated OL differentiation remains incompletely understood. We previously reported that genetically disrupting TCF712 inhibited OL differentiation with concomitant upregulation of the canonical bone morphogenetic protein (BMP)4 signaling in the CNS (Hammond et al., 2015), suggesting that the Wnt effector TCF7l2 may promote OL differentiation by tightly controlling BMP4 signaling, an inhibitory pathway of OL development (Grinspan, 2020). In this study, we unraveled a new mechanism of TCF7l2 in promoting OL differentiation by repressing autocrine BMP4 signaling.

\section{Materials and Methods \\ Animals}

The transgenic mice used in our study were: Olig2-CreER ${ }^{T 2}$ (Takebayashi et al., 2002), Plp-CreER ${ }^{T 2}$ (RRID: IMSR_JAX:005975), Pdgfr $\alpha$-CreER ${ }^{T 2}$ (RRID: IMSR_JAX:018280), Tcf7l2-floxed (RRID: IMSR_JAX:031436), Bmp4-floxed (RRID: IMSR_JAX:016878: the exon4 was flanked by two loxP sites). Cre transgene was always maintained as heterozygosity. Both male and female animals were used in the study. All animals were from C57BL/6 background and maintained in a 12/12 h light/dark cycle with water and food. Animal protocols were approved by the Institutional Animal Care and Use Committee at the University of California, Davis.

Gene conditional knock-out (cKO) by tamoxifen treatment

Tamoxifen (TM, T5648, Sigma) was dissolved in a mixture of ethanol and sunflower seed oil $(1: 9, \mathrm{v} / \mathrm{v})$ at $30 \mathrm{mg} / \mathrm{ml}$. All animals including $\mathrm{cKO}$ and control mice were intraperitoneally administered at a dose of $200 \mu \mathrm{g} / \mathrm{g}$ body weight.

\section{Magnetic-activated cell sorting (MACS)}

The forebrain was dissociated by the papain dissociation kit (LK003176, Worthington) and dissociator (Miltenyi Biotec, \#130-092-235). Astrocyte and microglia were purified by anti-ACSA-2 micro beads (130-097-679, Miltenyi Biotec) and anti-CD11b micro beads (130-049-601, Miltenyi Biotec), respectively. The cell suspension was then incubated with anti-O4 micro beads (130-094-543, Miltenyi Biotec) for OPC purification.

\section{Rodent primary culture of OPCs and differentiation}

Primary mouse OPCs were isolated from cortices of pups from postnatal day 0 (P0) to $\mathrm{P} 2$ using immunopanning procedure as described previously (Hammond et al., 2015; Zhang et al., 2018b, 2020). Cerebral cortices were enzymatically digested using papain $(20 \mathrm{U} / \mathrm{ml}$, \#LK003176, Worthington) supplemented with DNase I (250 U/ml; \#D5025, Sigma) and D-(+)-glucose $(0.36 \%$; \#0188 AMRESCO) and mechanically triturated to obtain single-cell suspension. The mixed glial cells were plated on poly-D-lysine (PDL, \#A003-E, Millipore)-coated 10-cm dishes and cultured in DMEM medium (\#1196092, Thermo Fisher Scientific) with $10 \%$ heat-inactivated fetal bovine serum (\#12306-C, Sigma) and penicillin/streptomycin (P/S; \#15140122, Thermo Fisher Scientific). After 24 h incubation, cells were washed using HBSS (with calcium and magnesium, \#24020117, Thermo Fisher Scientific), and cultured in serum-free growth medium (GM), containing 30\% of B104 neuroblastoma medium and $70 \%$ of N1 medium [DMEM with $5 \mu \mathrm{g} / \mathrm{ml}$ insulin (\#I6634, Sigma), $50 \mu \mathrm{g} / \mathrm{ml}$ apo-transferrin (\#T2036, Sigma), $100 \mu \mathrm{M}$ putrescine (\#P5780, Sigma), $30 \mathrm{~nm}$ Sodium selenite (\#S5261, Sigma), $20 \mathrm{~nm}$ progesterone (\#P0130, Sigma)] until 80\% of cellular confluency. The cells were then dissociated in single-cell suspension and seeded to Thy1.2 (CD90.2) antibody (\#105302, Biolegend)-coated Petri-dish to deplete astrocytes, neurons and meningeal cells followed by incubation on NG2 antibody (\#AB5320, Millipore)-coated Petri dish to select OPCs. OPCs were then cultured on PDL-coated plates with GM plus $5 \mathrm{ng} / \mathrm{ml}$ FGF (\#450-33, Peprotech), 4 ng/ml PDGF-AA (\#315-17, Peprotech), $50 \mu \mathrm{M}$ forskolin (\#6652995, Peprotech,) and glutamax (\#35050, Thermo Fisher Scientific). OPCs were differentiated in the differentiation medium, consisting of F12/high-glucose DMEM (\#11330032, Thermo Fisher Scientific) plus $12.5 \mu \mathrm{g} / \mathrm{ml}$ insulin, $100 \mu \mathrm{m}$ putrescine, $24 \mathrm{~nm}$ sodium selenite, $10 \mathrm{~nm}$ progesterone, $10 \mathrm{ng} / \mathrm{ml}$ biotin, $50 \mu \mathrm{g} / \mathrm{ml}$ transferrin (\#T8158, Sigma), $30 \mathrm{ng} / \mathrm{ml} \mathrm{3,3',5-triiodo-L-thyronine} \mathrm{( \# T5516,}$ Sigma), $40 \mathrm{ng} / \mathrm{ml}$ L-thyroxine (\#T0397, Sigma-Aldrich), glutamax, and $\mathrm{P} / \mathrm{S}$.

\section{Vector-mediated TCF7l2 overexpression in primary OLs and Oli-Neu} cells

The expression vectors pFlag-CMV2-TCF712-E2, pFlag-CMV2-TCF712S2, and pFlag-CMV2-TCF712-M1 were provided by Andreas Hecht at the University of Freiburg, Germany and were described in the original publication (Weise et al., 2010). For vector-mediated TCF712 overexpression in primary OPCs or Oli-Neu cells, the transfection was conducted using FuGENE6 kit (catalog \#2619, Promega) according to the manufacturer's protocol.

ELISA

The medium collected from primary culture were used for BMP4 measurement assay at day 2 of differentiation. The BMP 4 concentration was determined using the BMP4 ELISA kit (catalog \#OKEH02960, Aviva System Biology). ELISA was performed according to our previous protocol (Zhang et al., 2020).

\section{Chromatin immunoprecipitation (ChIP)}

ChIP assays were performed using a SimpleChIP Enzymatic Chromatin IP kit (\#9003; Cell Signaling Technologies). Briefly, cells were crosslinked using $37 \%$ formaldehyde at a final concentration of $1 \%$ for $10 \mathrm{~min}$ at room temperature and then quenched with $125 \mathrm{~mm}$ glycine for $5 \mathrm{~min}$. Cells were washed twice with cold PBS, harvested in PBS with protease inhibitor cocktail (PIC) and lysed to get nuclei. Chromatin was harvested, fragmented using micrococcal nuclease to length of $\sim 150$ $900 \mathrm{bp}$ and then briefly sonicated to lyse nuclear membranes. The digested chromatin $(500 \mu \mathrm{g})$ was subjected to IP with specific TCF7L2 antibody (Celling Signaling) or anti-rabbit IgG (\#2729, Cell Signaling) as negative control overnight at $4^{\circ} \mathrm{C}$ with rotation and followed by ChIPgrade protein-G magnetic beads incubated for $2 \mathrm{~h}$ at $4^{\circ} \mathrm{C}$ with rotation. After IP, the chromatin was eluted from the antibody/protein G magnetic beads and cross-links were reversed. DNA was purified and analyzed by RT-qPCR. Primer sequences (Integrated DNA Technologies) for the Bmp4 binding site were as follows: forward primer $5^{\prime}$ GGTACCTGCACTTAAGCTTTGTCGG-3' and reverse primer: $5^{\prime}$ TCGTAGTCGCTGCACGCAG-3' . The data were quantified by percent input and performed in triplicates.

\section{Protein extraction and Western blot assay}

Protein extraction and Western blot assay were performed as previously described with modifications (Wang et al., 2016; Zhang et al., 2020). Tissue or cells were lysed using N-PER Neuronal Protein Extraction Reagent (\#87792, Thermo Fisher Scientific) supplemented with PIC (\#5871S, Cell Signaling Technology) and phenylmethylsulfonyl fluoride (\#8553S, Cell Signaling Technology) on ice for $10 \mathrm{~min}$ and centrifuged at $10000 \times \mathrm{g}$ for $10 \mathrm{~min}$ at $4^{\circ} \mathrm{C}$ to pellet the cell debris. Supernatant was collected for concentration assay using BCA protein assay kit (\#23225, Thermo Fisher Scientific). Protein lysates $(30 \mu \mathrm{g})$ were separated by 
AnykD Mini-PROTEAN TGX precast gels (\#456-9035, BIO-RAD) or 7.5\% Mini-PROTEAN TGX precast gels (\#456-8024, Bio-Rad). The proteins were then transferred onto $0.2-\mu \mathrm{m}$ nitrocellulose membrane (\#1704158, Bio-Rad) using Trans-blot Turbo Transfer system (\#1704150, Bio-Rad). The membranes were blocked with 5\% BSA (\#9998, Cell Signaling) for $1 \mathrm{~h}$ at room temperature and incubated with primary antibodies at overnight $4^{\circ} \mathrm{C}$, followed by suitable horseradish peroxidase (HRP)-conjugated secondary antibodies. Proteins of interest were detected using Western Lightening Plus ECL (NEL103001EA, PerkinElmer). NIH ImageJ was used to quantify protein levels by analyzing the scanned gray-scale films. Primary and secondary antibodies used were: TCF712 (1:1000, RRID:AB_2199816, \#2569S, Cell Signaling Technology), MBP (1:1000, RRID:AB_2139899, \#NB600-717, Novus), CNPase (1:1000, RRID:AB_2082474, \#2986, Cell Signaling Technology), histone deacetylase 2 (HDAC2; 1:1000, RRID:AB_10624871, \#5113, Cell Signaling Technology), TLE3 (1:1000, RRID:AB_2203743, \#11 3721-AP, Proteintech), $\beta$-actin (1:1000, RRID:AB_330288, \#4967L, Cell Signaling Technology), GAPDH (1:1000, RRID:AB_561053, \#2118L, Cell Signaling Technology), and HRP goat anti-rabbit (1:3000, RRID:AB_ 228341, \#31460, Thermo Fisher Scientific), anti-mouse (1:3000, RRID: AB_228307, \#31 430, Thermo Fisher Scientific), or anti-rat (1:3000, RRID: AB_10694715, \#7077, Cell Signaling Technology) secondary antibodies.

\section{Protein Co-IP}

Co-IP was performed as previously described with some modifications (Lang et al., 2013; Wang et al., 2017). Proteins were extracted by Pierce IP lysis/wash buffer (\#87787, Thermo Fisher Scientific), and protein concentration was evaluated using BCA protein assay kit; $1 \mathrm{mg}$ of proteins were incubated with TCF712 antibody (\#2569S, Cell Signaling Technology) or normal rabbit IgG (\#2729, Cell Signaling Technology) as negative control for IP overnight at $4^{\circ} \mathrm{C}$. Pierce Protein A/G Magnetic Beads (\#88802, Thermo Fisher Scientific) were washed three times with Pierce IP lysis/ wash buffer and added to antigen/antibody complex for $1 \mathrm{~h}$ at room temperature. Beads were then washed twice with IP lysis/wash buffer and once with purified water. The antigen/antibody complex was eluted from beads by boiling in Lane Marker Sample buffer (\#39001, Thermo Fisher Scientific) containing $\beta$-mercaptoethanol for SDS-PAGE and Western blotting.

\section{$R N A$ extraction, $c D N A$ preparation, and $R T-q P C R$ assay}

Total RNA was isolated by RNeasy Lipid Tissue Mini kit (\#74804, QIAGEN) with on-column DNase I digestion to remove genomic DNA using RNase-Free DNase Set (\#79254, QIAGEN). The concentration of RNA was determined by Nanodrop 2000. cDNA was synthesized by QIAGEN Omniscript RT kit (\#205111, QIAGEN). RT-qPCR was performed by using QuantiTect SYBR Green PCR kit (\#204145, QIAGEN) on Agilent MP3005P thermocycler. The relative mRNA level of indicated genes was normalized to that of the internal control gene Hsp90 (Hsp90ab1), using the equation 2a(Ct(cycle threshold) of Hsp90 - Ct of indicated genes) according to our previous protocol (Hull et al., 2020). The gene expression levels in control groups were normalized to 1 . The RT-qPCR primers used in this study were as followed:

Sox10 (F: ACACCTTGGGACAC GGTTTTC, R: TAGGTCTTGTTC CTCGGCCAT),

Enpp6 (F: CAGAGAGATTGTGA ACAGAGGC, R: CCGATCATCT GGTGGACCT),

Mbp (F: GGCGGTGACAGACT CCAAG, R: GAAGCTCGTCGG ACTCTGAG),

Plp (F: GTTCCAGAGGCCAACATCAAG, R: CTTGTCGGGATG TCCTAGCC),

Mog (F: AGCTGCTT CCTCTCCCTTCTC, R: ACTAAAGCCCGG ATGGGATA C),

Myrf (F: CAGACCCAGGTGCTACAC, R: TCCTGCTTGATCATT CCGTTC),

Bmp4-exon4 (F: TGATCACCTCAACTCAACCAAC, R: TCAT CCAGGTACAACATGGAAA),

Tcf7l2 (F: GGAGGAGAAGAACTCGGAAAA, R: ATCGGAGG AGCTGTTTTGATT),
Axin2 (F: AACCTATGCCCGTTTCCTCTA, R: GAGTGTAAA GACTTGGTCCACC),

Naked1 (F: CAGCTTGCTGCATACCATCTAT, R: GTTGAAAAG GACGCTCCTCTTA),

Sp5 (F: GTACTTGCCATCGAGGTAG， R: GGCTCGGACTTT GGAATC),

Gfap (GTGTCAGAAGGCCACCTCAAG/CGAGTCCTTAATGA CCTCACCAT),

Slc1a2 (CAACGGAGGATATCAGTCTGC/TGTTGGGAGTCAAT GGTGTC),

Id1 (F: CTCAGGATCATGAAGGTCGC, R: AGACTCCGAGTT CAGCTCCA),

Id2 (F: ATGGAAATCCTGCAGCACGTC, R: TGGTTCTGT CCAGGTCTCT),

Id3 (CGACCGAGGAGCCTCTTAG/GCAGGATTTCCACCTGG CTA),

Id4 (CAGTGCGATATGAACGACTGC/GACTTTCTTGTTGGGC GGGAT),

Hsp90 (F: AAACAAGGAGATTT TCCTCCGC, R: CCGTCAGGCT CTCATATCGAAT).

cRNA probe preparation, $m R N A$ in situ hybridization (ISH), and combined $m R N A$ ISH and immunohistochemistry (ISH/IHC)

Probe preparation, mRNA ISH, and combination of ISH/IHC were performed by using the protocols in our previous studies (Hammond et al., 2015; Zhang et al., 2020). In brief, we used a PCR-based approach to amplify 907- and 623-bp fragments of Bmp4 and Mag mRNA, respectively. The primer sets we used were: $B m p 4$ forward: $5^{\prime}$-CCTGCA GCGATCCAGTCT-3'/Bmp4 reverse: 5' -GCCCAATCTCCACTCCCT3'; Mag forward: 5' -CAAGAGCCTCTACCTGGATCTG-3'/Mag reverse: 5'-AGGTTCCTGGAGGTACAAATGA-3'. The core sequence (GCG ATTTAGGTGACACTATAG) recognized by SP6 RNA Polymerase was attached to the $5^{\prime}$ of the reverse primers. The core sequence (GCGT AATACGACTCACTATAGGG) recognized by T7 RNA polymerase was attached to the $5^{\prime}$ of the forward primers. The resulting PCR products were purified and used as templates for in vitro transcription by employing DIG RNA Labeling kit (SP6/T7; Roche \#11175025910). The resulting RNAs generated from SP6-mediated in vitro transcription were used as positive probes (cRNA, complementary to the target mRNAs), whereas those generated from T7-mediated in vitro transcription were used as negative probes (same sequence as the target mRNA). Frozen sections $(12 \mu \mathrm{m}$ thick) were used for mRNA ISH. Hybridization was performed in a humidity chamber at $65^{\circ} \mathrm{C}$ for $12-18 \mathrm{~h}$, followed by a series of stringent washing by saline-sodium citrate (SSC) buffer at $65^{\circ} \mathrm{C}(2 \times$ SSC $20 \mathrm{~min}$ for three times and then $0.2 \times$ SSC $20 \mathrm{~min}$ for three times) and RNase digestion at $37^{\circ} \mathrm{C}(30 \mathrm{~min})$ to eliminate non-specific binding and non-hybridized mRNAs. The DIG signals were visualized by NBT/BCIP method (Roche, \#11697471001). Depending on the abundance of target mRNAs, the duration of NBT/BCIP incubation $\left(\right.$ at $4^{\circ} \mathrm{C}$ ) ranged from $4 \mathrm{~h}$ to overnight.

For combined ISH/IHC, frozen sections (12 $\mu \mathrm{m}$ thick) were used. DIG-conjugated cRNA probe hybridization was performed according to the procedure described above. After a series posthybridization SSC washing and RNase digestion, the sections were briefly rinsed in TS7.5 buffer $(0.1 \mathrm{M}$ Tris- $\mathrm{HCl}$ and $0.15 \mathrm{M} \mathrm{NaCl} ; \mathrm{pH} 7.5)$ followed by incubation in $1 \%$ blocking reagent (prepared in TS7.5 buffer; Roche \#11096176001) for $1 \mathrm{~h}$ at room temperature. After blocking, a cocktail of anti-Sox10 antibody (1:100, \#AF2864, R\&D Systems) and AP-conjugated anti-DIG antibody (Roche \#11093274910) diluted in 1\% blocking reagent was applied and incubated at $4^{\circ} \mathrm{C}$ for overnight. The sections were then washed in TNT buffer (TS7.5 $+0.05 \%$ Tween 20) $10 \mathrm{~min}$ for three times. The primary antibody of Sox10 was visualized by incubating Alexa Fluor 488-conjugated secondary antibody at room temperature for $1 \mathrm{~h}$. The DIG-conjugated mRNA signals were visualized by HNPP/Fast Red Fluorescent Detection set (Roche \#11758888001) according to the kit instructions.

Tissue preparation and IHC

Tissue preparation and IHC were conducted as previously described (Hammond et al., 2015; Zhang et al., 2018b, 2020). Mice were perfused 
with ice-cold PBS (\#BP399-20, Fisher Chemical), and postfixed in fresh prepared 4\% paraformaldehyde (PFA; \#1570-S, Electron Microscopy Science) at room temperature for $2 \mathrm{~h}$. Tissues were washed three times (15 min per each time) using ice-cold PBS and cryoprotected using 30\% sucrose (\#S5-3, Fisher Chemical) in PBS at $4^{\circ} \mathrm{C}$ overnight followed by embedding in OCT prior. Twelve-micrometer-thick sections were serially collected and stored in $-80^{\circ} \mathrm{C}$. For IHC, slice was air dry in room temperature for at least $1 \mathrm{~h}$ and blocked with $10 \%$ donkey serum in $0.1 \%$ Triton X-100/PBS (v/v) for $1 \mathrm{~h}$ at room temperature. Tissue was incubated with primary antibodies overnight at $4^{\circ} \mathrm{C}$, washed three times in PBST (PBS with $0.1 \%$ Tween 20), and incubated with secondary antibodies at room temperature for $2 \mathrm{~h}$. DAPI was applied as nuclear counterstain. All images shown were obtained by Nikon C1 confocal microscope. An optical thickness of $10 \mu \mathrm{m}$ was used for confocal z-stack imaging (step size $1 \mu \mathrm{m}$ and 11 optical slices), and the maximal projection of the $\mathrm{z}$-stack images was used for data quantification. Primary antibodies included Sox10 (1:100, RRID: AB_442208, \#AF2864, R\&D Systems), Sox10 (1:100, RRID:AB_ 2650603, \#ab155279, Abcam), HDAC2 (1:100, RRID:AB_365273, \#5113P, Cell Signaling Technology), TCF712 (1:100, RRID:AB_ 2199816, \#2569S, Cell Signaling Technology), $\beta$-catenin (1:200, RRID:AB_476865, \#7202, Sigma), P-Smad 1/5 (1:200, RRID:AB_ 491015, \#9516, Cell Signaling Technology), CC1 (1:100, RRID:AB_ 2057371, \#OP80, Millipore), PDFGR $\alpha$ (1:100, RRID:AB_2236897, \#AF1062, R\&D Systems), adenomatous polyposis coli (APC; 1:100, RRID:AB_2057493, sc-896, Santa Cruz Biotechnology), BLBP (1:100, RRID:AB_10000325, \#ABN14, Millipore), and Sox9 (1:200, RRID:AB_ 2194160,\#AF3075, R\&D Systems). All species-specific secondary antibodies (1:500) were obtained from Jackson ImmunoResearch.

\section{Immunocytochemistry (ICC)}

Primary cells attached to the coverslips were fixed in $4 \%$ PFA for $15 \mathrm{~min}$ followed by rinse with PBS. The ICC was performed as our previous studies (Guo et al., 2012; Hammond et al., 2015; Zhang et al., 2020). The following antibodies were used in our study: Sox10 (1:100, RRID: AB_ 2195374, \#sc-17342, Santa Cruz Biotechnology; 1:500, RRID:AB_ 778021, \#ab27655, Abcam), Mbp (1:500, RRID:AB_2564741, \#SMI-99, Biolegend), TCF712 (1:200, RRID:AB_2199826, \#2569S, Cell Signaling Technology), P-Smad1/5 (1:200, RRID:AB_491015, \#9516, Cell Signaling Technology).

\section{Gene clone and luciferase activity assay}

To generate a luciferase reporter under the control of the intronic promoter of the mouse Bmp4 gene, a 439-bp DNA fragment containing the Bmp4 promoter was amplified using the spinal cord genomic DNA with the forward primer 5'-GGTACCTGCACTTAAGCTTTGTCGG - $3^{\prime}$ (underlined sequence is $\mathrm{KpnI}$ site, bold italic sequence is TCF7l2 binding site) and the reverse primer $5^{\prime}$-CTCGAGACGGAATGGCTCCTAA AATG-3' (underlined sequence is Xhol site). The PCR product was cloned into pGEM-T-Easy vector and confirmed by DNA sequencing. After digesting with KpnI and XhoI, the fragment was cloned into pGL2Basic vector. The resulting luciferase reporter was designated as pGL2Bmp4-Luc. Using pGL2-Bmp4-Luc as a template, TCF712-binding mutation constructs were generated by PCR using the above reverse primer and the following forward primer: GGTACCTGCACTTAAGTGG TGTCGG (underlined sequence is KpnI site). The resulting mutated luciferase reporter was designated as pGL2-mBmp4-Luc. The dual luciferase assay was done in triplicate using TD-20/20Luminometer (Promega) according to the manufacturer's instructions. Briefly, $0.25 \mu \mathrm{g}$ of a luciferase reporter (Bmp4-Luc or mutBmp4-Luc), $0.25 \mu \mathrm{g}$ of TCF712-S2 or TCF712-E2, and $5 \mathrm{ng}$ of Renilla luciferase report (Promega) were co-transfected into U87 human primary glioblastma cells by using ESCORT V transfection reagent (Sigma). The fold increase in relative luciferase activity is a product of the luciferase activity induced by TCF712-S2 or TCF712-E2 (backbone vector $\mathrm{pCMV}$ ) divided by that induced by an empty $\mathrm{pCMV}$ vector.

Experimental designs and statistical analyses

All quantification was conducted by lab members blinded to genotypes or treatments. For in vivo gene cKO studies, animals were derived from multiple litters. Data were presented as mean \pm SEM throughout this manuscript. We used bar graph and scatter dot plots to show the distribution of the data. Each dot (circle, square, or triangle, if applicable) in the scatter dot plots represents one mouse or one independent experiment. We used Shapiro-Wilk approach for testing data normality. $F$ test and Browne-Forsythe test were used to compare the equality of variances of two and three or more groups, respectively. The statistical analysis details of Figures 2-7 were summarized in Table 1; $p$ values of $t$ test were displayed in each graph. Welch's correction was used for Student's $t$ test where the variances of two groups were unequal. For comparisons among three or more groups with equal variances, ordinary one-way ANOVA was used followed by Tukey's multiple comparisons, otherwise Welch's ANOVA was used followed by unpaired $t$ test with Welch's correction. All data plotting and statistical analyses were performed using GraphPad Prism version 8.0; $p<0.05$ was considered as significant, whereas $p>0.05$ was assigned as not significant (ns).

\section{Results}

BMP4 is expressed primarily in oligodendroglial lineage cells BMP4 is a secreted protein and the mRNA expression better reflects its cellular origin. To determine cellular source of BMP4 in vivo, we purified different cell populations from the brain by MACS (Fig. $1 A$ followed by mRNA quantification). We found that BMP4 was highly enriched in $\mathrm{O}^{+}$oligodendroglial lineage (Fig. 1B), which consists of late postmitotic OPCs and immature OLs (Armstrong et al., 1992). To determine BMP4 dynamics within the oligodendroglial lineage, we employed primary OPC culture and differentiation system (Zhang et al., 2018b) and demonstrated that BMP4 was low in OPCs and rapidly upregulated (by $>150$-fold) in differentiating OLs (Fig. 1C), a dynamic pattern correlated with that of TCF712 (Fig. 1D; Hammond et al., 2015), suggesting a potential link between BMP4 and TCF7l2 during OL differentiation.

\section{TCF712 deletion upregulates BMP4 and activates canonical BMP4 signaling in vivo}

Our prior study reported an upregulation of BMP4 in the CNS of TCF712 cKO mutants (Hammond et al., 2015). To determine the regulation of BMP4 at the cellular level, we deleted TCF712 in the oligodendroglial lineage using time-conditioned Olig2$\mathrm{CreER}^{\mathrm{T} 2}$ and assessed BMP4 expression in vivo $24 \mathrm{~h}$ after tamoxifen treatment (Fig. 2A). During this short time window, TCF712 deletion-elicited OL differentiation defect (Hammond et al., 2015; Zhao et al., 2016) was not dramatically prominent, as indicated by $\sim 28 \%$ decrease in the cell number of mRNA ISH for the mature OL marker MAG (Fig. $2 B$ ). We found a significant increase of Bmp4 mRNA in TCF712 cKO spinal cord demonstrated by RT-qPCR (Fig. 2C) and confirmed by ISH (Fig. 2D). Combined ISH and IHC showed that BMP4 was activated in Sox $10^{+}$cells in the spinal cord of TCF712 cKO mice (Fig. 2E), indicating that TCF712 represses oligodendroglial BMP4 expression in vivo.

BMP4 activates canonical BMP signaling through phosphorylating SMAD1/5 (Retting et al., 2009), thus the phosphorylated form of SMAD1/5 (p-SMAD1/5) is a reliable surrogate for the signaling activation. We next used IHC antibodies specifically recognizing $\mathrm{p}$-SMAD1/5 to assess BMP signaling activation at the cellular level. We found that $\mathrm{p}-\mathrm{SMAD} 1 / 5$ was primarily observed in Sox10-negative cells, presumably astroglial lineage cells in Tcf7l2 Ctrl mice (Fig. $2 F$, arrows), suggesting that, under normal condition, the activity of BMP/SMAD signaling is minimal in oligodendroglial lineage cells. In contrast, p-SMAD1/5 immunoreactive signals were consistently observed in Sox10expressing oligodendroglial cells (Fig. 2F, arrowheads) in TCF7l2 
A

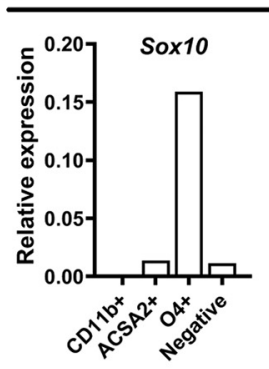

Oligodendroglial markers

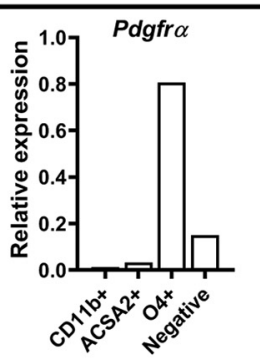

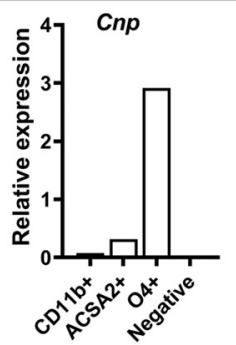

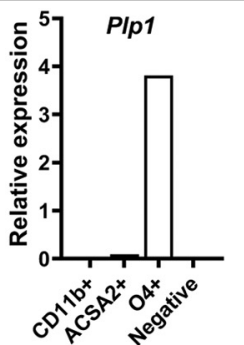

\section{Neuronal marker}

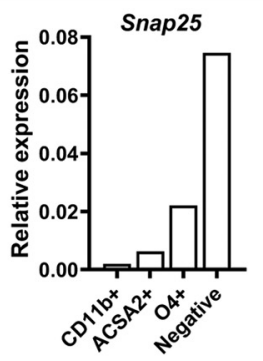

Microglial markers
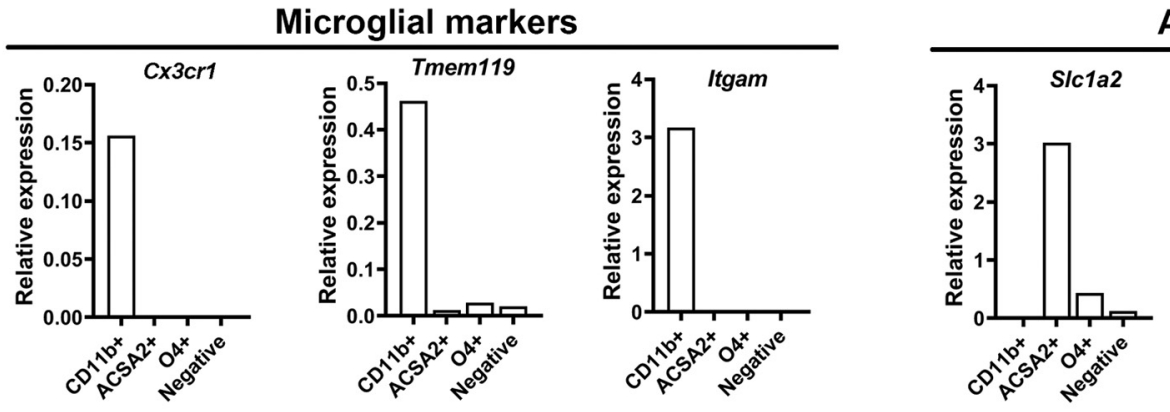

Astroglial markers
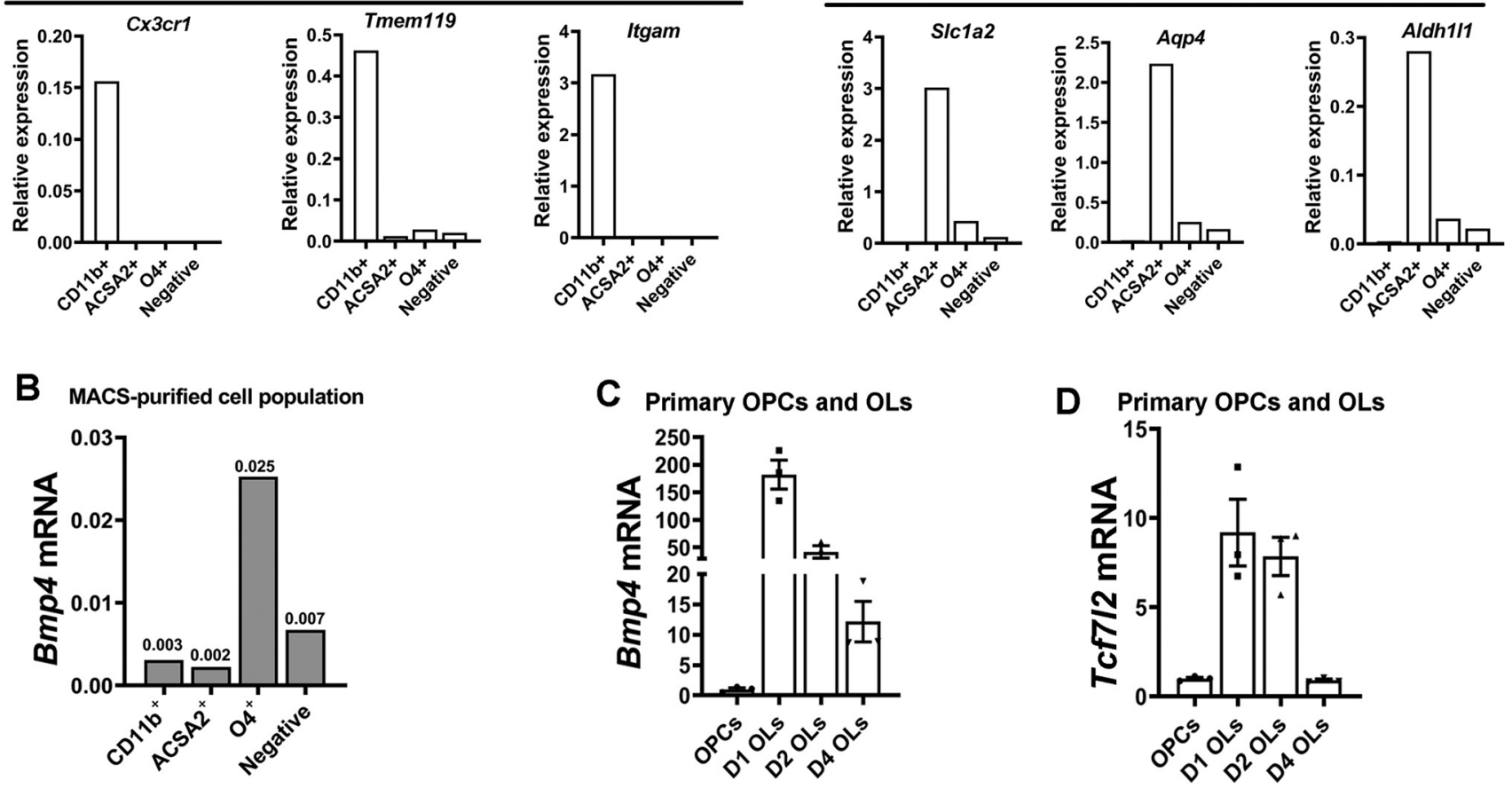

Figure 1. BMP4 is expressed primarily in newly differentiated OLs. The brain of P5 C57BL/6 wild-type mice ( $n=3$, pooled together) was used for cell purification by MACS. CD11b, ACSA2, and 04-conjugated magnetic beads were used for positively selecting microglial, astroglial, and oligodendroglial lineage cells, respectively. Brain cells negative for CD11b, ACSA2, and 04 were

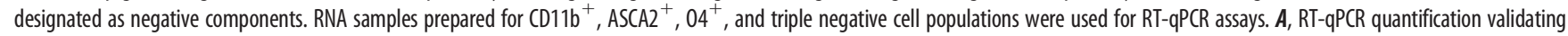
the efficacy of MACS purification of each cell populations by lineage-specific markers (normalized to the internal control gene Hsp90 in each population). $\boldsymbol{B}$, RT-qPCR assay for Bmp4 in MACSpurified brain microglial $\left(\mathrm{CD} 11 \mathrm{~b}^{+}\right)$, astroglial $\left(\mathrm{ACSA}^{+}\right)$, and oligodendroglial lineage $\left(04^{+}\right)$cells at P5. C, $\boldsymbol{D}, \mathrm{RT}-\mathrm{qPCR}$ assay for Bmp4 $(\boldsymbol{C})$ and $T c f 7 / 2(\boldsymbol{D})$ in primary rodent $0 \mathrm{PCS}$ maintained in proliferating medium and differentiating 0Ls maintained in differentiation medium for 1-4 d (D1-D4 0Ls). D1-D2 0Ls, immature 0Ls; D4 0Ls, mature 0Ls.

cKO mice. We noticed that $\mathrm{p}-\mathrm{SMAD}^{+}$oligodendroglial lineage cells in Tcf7l 2 cKO mice displayed weak Sox10 expression and relatively small $\mathrm{DAPI}^{+}$nuclei, which is characteristic feature of OPCs and differentiating immature OLs reported by Reiprich and colleagues (Reiprich et al., 2017). Furthermore, the expression of canonical BMP signaling target genes $I d 1, I d 2$, and $I d 3$ was significantly elevated in TCF7l2 cKO mice (Fig. 2G). These data suggest that TCF712 normally represses BMP4/SMAD signaling and its conditional depletion results in the activation of the signaling in oligodendroglial lineage cells. The upregulation of BMP4 and the signaling activity were confirmed in the brain by an independent $P l p 1-C r e E R^{\mathrm{T} 2}, T c f 7 l 2^{\mathrm{fl} / \mathrm{fl}}$ mutant mice (Fig. $2 \mathrm{H}-\mathrm{K})$, suggesting that TCF7l2 controls BMP4 and the canonical BMP4 signaling activity in an autocrine manner not only in the spinal cord but also in the brain.

BMP4 enhances astrocyte generation at the expense of oligodendrogenesis (Gomes et al., 2003; Wu et al., 2012; Grinspan, 2020). To determine the effect of BMP4 upregulation elicited by TCF712 cKO on astrocyte development, we quantified astrocyte density in vivo. No significant difference was observed in the number of Sox $9^{+} \mathrm{BLBP}^{+}$or $\operatorname{Sox} 9^{+} \mathrm{S} 100 \beta^{+}$astrocytes (Fig. $3 A-D)$, nor the expression of mature astrocyte markers GFAP and GLT-1 (Fig. 3E,F) between TCF7l2 Ctrl and cKO mice. Collectively, our data demonstrate that the Wnt effector TCF7l2 represses BMP4 expression and controls autocrine BMP signaling activity during OL differentiation.

\section{TCF712 directly represses BMP4 transcription}

A repressive role of TCF712 in BMP4 has not been reported in oligodendroglial lineage cells. Bioinformatic analysis showed that the intronic promoter of the mouse Bmp4 gene (Thompson et al., 2003) contained a putative DNA binding motif of TCF712 (Fig. 4A). ChIP followed by qPCR (ChIP-qPCR) confirmed the physical binding of TCF7l2 to this regulatory element (Fig. $4 B$ ). To determine the functional output of TCF712 binding, we sought to analyze the effect of TCF7l2 overexpression on the intronic Bmp4 promoter-driven luciferase activity. 
Table 1. Detailed information of statistical analyses

\begin{tabular}{|c|c|c|}
\hline Figure & Sample size $(n)$ & Statistical methods, $t_{\text {(df) }}$ value, $F$ values \\
\hline $2 B$ & $\begin{array}{l}n=4 \text { TCF7l2 Ctrl } \\
n=3 \text { TCF7l2 cKO }\end{array}$ & Two-tailed Student's $t$ test, $t_{(5)}=2.937$ \\
\hline $2 C$ & $\begin{array}{l}n=4 \text { TCF7l2 Ctrl } \\
n=3 \text { TCF7I2 CKO }\end{array}$ & Two-tailed Student's $t$ test, $t_{(5)}=5.582$ \\
\hline $2 G$ & $\begin{array}{l}n=4 \text { TCF7l2 Ctrl } \\
n=3 \text { TCF7I2 CKO }\end{array}$ & Two-tailed Student's $t$ test, Welch-corrected $t_{(2.032)}=7.729 \mathrm{Id} 1, t_{(5)}=2.910 \mathrm{Id} 2, t_{(5)}=3.744 \mathrm{Id} 3$ \\
\hline $21-K$ & $\begin{array}{l}n=4 \text { TCF7l2 Ctrl } \\
n=3 \text { Plp-CreERT2:TCF712 cK0 }\end{array}$ & Unpaired, two-tailed Student's $t$ test, Welch-corrected $t_{(5.576)}=7.833 \mathrm{Tcf} .712, t_{(10)}=11.08 \mathrm{Bmp} 4, t_{(10)}=3.513 \mathrm{Id} 3$. \\
\hline $3 E, F$ & $\begin{array}{l}n=5 \mathrm{TCF} 72 \mathrm{Ctrl} \\
n=5 \mathrm{TCF} 72 \mathrm{CKO}\end{array}$ & Two-tailed Student's $t$ test, $t_{(8)}=0.1488$ Gfap, $t_{(8)}=0.2492$ Slc1a2 \\
\hline $4 B$ & $n=3 \operatorname{lgG}, n=3 \mathrm{TCF} 7 \mathrm{I} 2$ & Two-tailed Student's $t$ test, $t_{(4)}=14.00$ \\
\hline $4 G$ & $n=3$ each condition & One-way ANOVA, followed by Tukey's post hoc test $F_{(2,6)}=11.69, p=0.0085 ;{ }^{*} p<0.05,{ }^{* *} p<0.01$, \\
\hline $4 H$ & $n=3$ each condition & One-way ANOVA, followed by Tukey's post hoc test, $p=0.0058 ;{ }^{*} p<0.05$, ** $p<0.01$, \\
\hline $5 E$ & $n=3 \mathrm{EV}, n=3 \mathrm{TCF} 7 \mathrm{I} 2$ & Two-tailed Student's $t$ test, $t_{(4)}=2.960 \mathrm{Mbp}, t_{(4)}=4.458 \mathrm{Plp}, t_{(4)}=3.464 \mathrm{Mog}, t_{(4)}=4.303 \mathrm{Enpp} 6$, and $t_{(4)}=4.976 \mathrm{Myrf}$ \\
\hline $5 F$ & $n=3 \mathrm{EV}, n=3 \mathrm{TCF} 7 \mathrm{I}$ & Two-tailed Student's $t$ test, $t_{(4)}=4.631$ \\
\hline $5 G$ & $n=3 \mathrm{EV}, n=3 \mathrm{TCF} 7 \mathrm{I}$ & Two-tailed Student's $t$ test, $t_{(4)}=2.839$. \\
\hline $5 H$ & $n=3 \mathrm{EV}, n=3 \mathrm{TCF} 7 \mathrm{I}$ & Two-tailed Student's $t$ test, $t_{(4)}=4.976$ \\
\hline 51 & $n=3 \mathrm{EV}, n=3 \mathrm{TCF} 7 \mathrm{I} 2$ & Two-tailed Student's $t$ test, $t_{(4)}=0.1368$ Axin2, $t_{(4)}=1.724 \mathrm{Nkd1}$, and $t_{(4)}=1.622 \mathrm{Sp5}$. \\
\hline $5 K$ & $n=3 \mathrm{Ctrl} n=3 \mathrm{BMP} 4$ & Two-tailed $t$ test, Welch's corrected $t_{(2.009)}=9.529$. \\
\hline $5 L$ & $n=3$ each condition & $\begin{array}{l}\text { One-way ANOVA, followed by Tukey's post hoc test. } \\
F_{(3,8)}=25.82, p=0.0002 .{ }^{* *} p<0.01,{ }^{* * *} p<0.001\end{array}$ \\
\hline $6 B$ & $\begin{array}{l}n=13 \text { control } \\
n=4 \text { TCF7I2 cK0 } \\
n=4 \text { TCF7I2/BMP4 cK0 }\end{array}$ & One-way ANOVA, followed by Tukey's post hoc test, $F_{(2,18)}=47.01 p<0.0001$; *** $p<0.001$, ns, not significant \\
\hline $6 C$ & $\begin{array}{l}n=13 \text { control } \\
n=4 \text { TCF7I2 cK0 } \\
n=4 \text { TCF7I2/BMP4 cK0 }\end{array}$ & One-way ANOVA, followed by Tukey's post hoc test, $F_{(2,18)}=87.15 p<0.0001$; ** $p<0.001$ \\
\hline $6 H$ & $\begin{array}{l}n=11 \text { control } \\
n=4 \text { TCF7I2 cKO } \\
n=4 \text { TCF7I2/BMP4 CKO }\end{array}$ & One-way ANOVA, followed by Tukey's post hoc test, $F_{(2,16)}=38.82, p<0.0001 ;{ }^{*} p<0.05,{ }^{* * *} p<0.001$ \\
\hline $6 J$ & $\begin{array}{l}n=3 \text { control } \\
n=3 \text { TCF7I2 cK0 } \\
n=3 \text { TCF7I2/BMP4 cK0 }\end{array}$ & One-way ANOVA, followed by Tukey's post hoc test, $F_{(2,6)}=21.30, p=0.0019 ;{ }^{*} p<0.05,{ }^{* *} p<0.01$ \\
\hline $6 K$ & $\begin{array}{l}n=3 \text { control } \\
n=3 \text { TCF7I2 cK0 } \\
n=3 \text { TCF7I2/BMP4 cK0 }\end{array}$ & One-way ANOVA, followed by Tukey's post hoc test, $F_{(2,6)}=13.55, p=0.0060 ;{ }^{*} p<0.05,{ }^{* *} p<0.01$ \\
\hline $6 M$ & $\begin{array}{l}n=5 \text { control } \\
n=4 \text { TCF7I2 cKO } \\
n=5 \text { TCF7I2/BMP4 cKO }\end{array}$ & $\begin{array}{l}\text { One-way ANOVA, followed by Tukey's post hoc test, } F_{(2,11)}=107.9 p<0.0001 \text { Sox } 10^{+}, F_{(2,11)}=99.72 p<0.00010 \mathrm{Ls}, F_{(2,11)}=2.945 \\
\quad p=0.0946 \text { OPCs; }{ }^{* *} p<0.01,{ }^{* * * *} p<0.0001 \text {, ns, not significant }\end{array}$ \\
\hline $6 \mathrm{~N}$ & $\begin{array}{l}n=5 \text { control } \\
n=4 \text { TCF7I2 cKO } \\
n=5 \text { TCF7I2/BMP4 cKO }\end{array}$ & $\begin{array}{l}\text { One-way ANOVA, followed by Tukey's post hoc, } F_{(2,11)}=23.70 p=0.0001 \text { Sox } 10^{+}, F_{(2,11)}=29.91 p<0.00010 \mathrm{Ls}, F_{(2,11)}=0.4095 \\
\quad p=0.6737 \mathrm{OPCS} ;{ }^{* * *} p<0.001 \text {, ns, not significant }\end{array}$ \\
\hline $7 A$ & $\begin{array}{l}n=3 \text { BMP4 Ctrl } \\
n=4 \text { BMP4 CKO }\end{array}$ & Two-tailed Student's $t$ test, $t_{(5)}=15.29$. \\
\hline $7 C$ & $\begin{array}{l}n=3 \text { BMP4 Ctrl } \\
n=4 \text { BMP4 cKO }\end{array}$ & Two-tailed Student's $t$ test, $t_{(5)}=1.7180 \mathrm{Ls}, t_{(5)}=1.8290 \mathrm{PCs}$ \\
\hline $7 D$ & $\begin{array}{l}n=3 \text { BMP4 Ctrl } \\
n=4 \text { BMP4 cKO }\end{array}$ & Two-tailed Student's $t$ test, $t_{(5)}=0.96460 \mathrm{Ls}, t_{(5)}=1.0880 \mathrm{PCs}$ \\
\hline $7 E$ & $\begin{array}{l}n=3 \text { BMP4 Ctrl } \\
n=4 \text { BMP4 CKO }\end{array}$ & Two-tailed Student's $t$ test, $t_{(5)}=0.0817 \mathrm{Mbp}, t_{(5)}=0.4940 \mathrm{Plp}$, and $t_{(5)}=0.2660$ Sox 10 \\
\hline $7 G, H$ & $\begin{array}{l}n=3 \text { BMP4 Ctrl } \\
n=4 \text { BMP4 CKO }\end{array}$ & Two-tailed Student's $t$ test, $t_{(5)}=0.8275$ white matter, $t_{(5)}=0.7165$ gray matter \\
\hline
\end{tabular}


Table 1. Continued

\begin{tabular}{lll}
\hline Figure & Sample size $(n)$ & Statistical methods, $t_{(\mathrm{df})}$ value, $F$ values \\
\hline $7 I$ & $n=3$ BMP4 Ctrl & Two-tailed Student's $t$ test, $t_{(5)}=0.4053$. \\
$7 J$ & Two-tailed Student's $t$ test, $t_{(5)}=1.259$ Id1, $t_{(5)}=0.5821$ Id2. \\
& $n=3$ BMP4 Ctrl \\
& $n=4$ BMP4 cK0 & \\
\hline
\end{tabular}

A

\section{OCE-Tcf7I2 cKO \\ Tcf7l2 Ctrl}

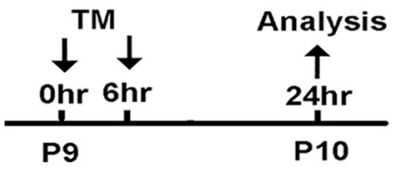

B

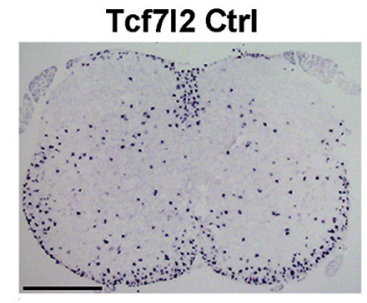

OCE-Tcf7I2 cKO

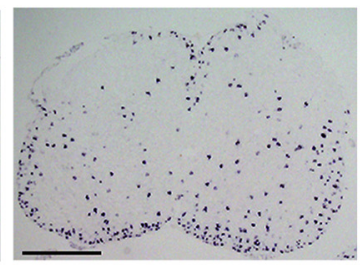

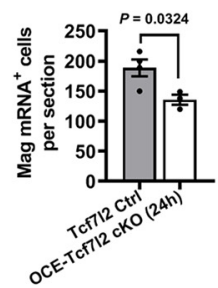

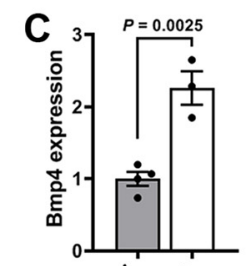
$112 \mathrm{Ctrl}(24 n)$ CE CAF CF
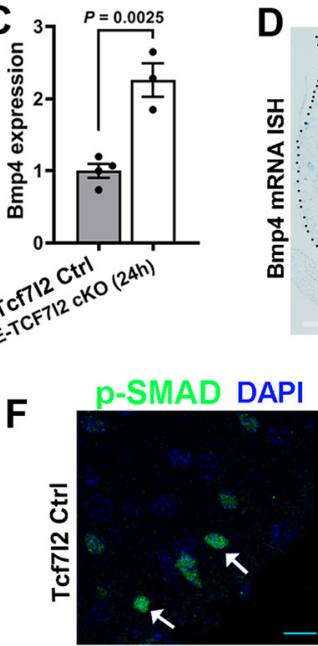

Tcf7I2 Ctr

OCE-Tcf7I2 cKO
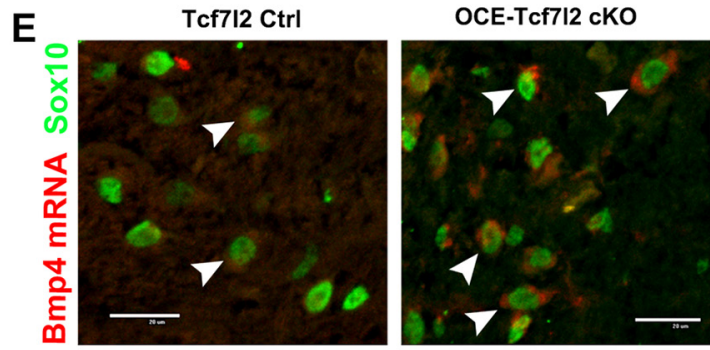
$\square$ Tcf7l2 Ctrl $\square$ OCE-Tcf7|2 cKO
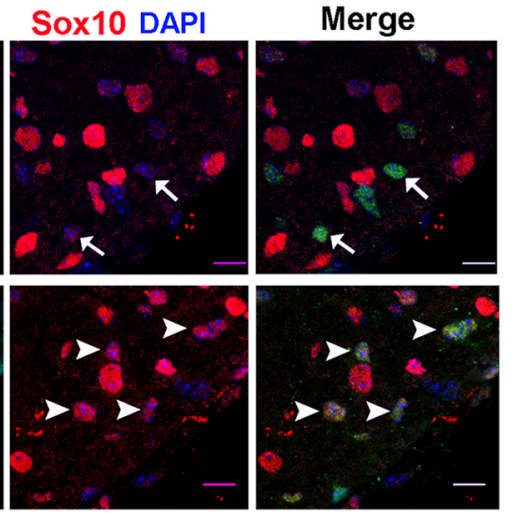

G

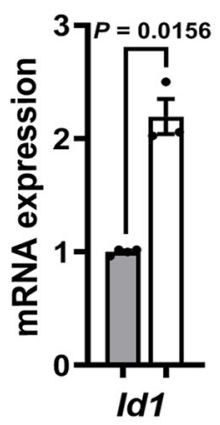

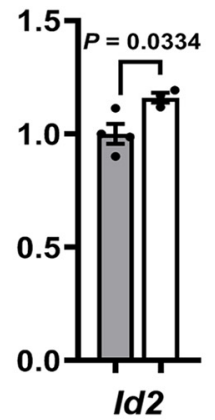

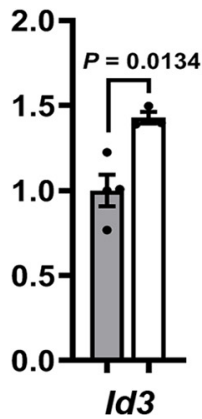

$\square$ Tcf7l2 Ctrl $\square$ PIp-CreERT2:TCF7|2 cKO
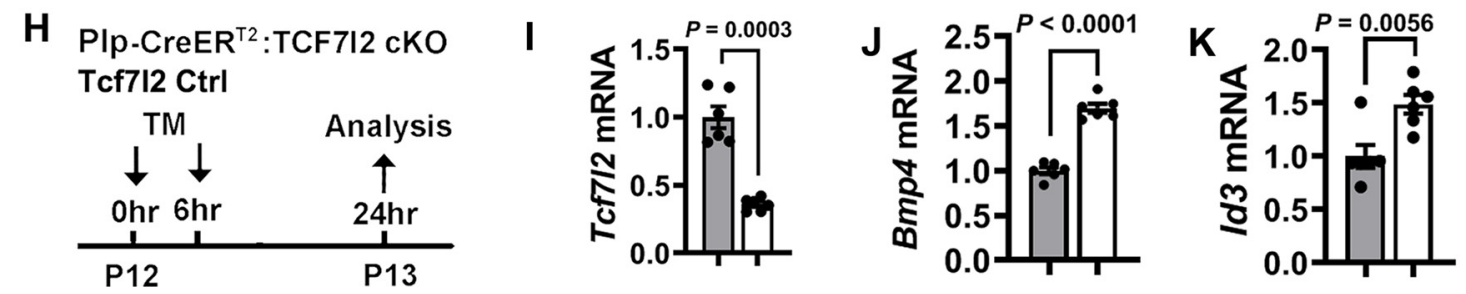

Figure 2. TCF7I2 deletion activates BMP4 and BMP4-mediated signaling in oligodendroglial lineage. A, Experimental design for B-G. OCE-Tcf7l2 CKO (Olig2-CreER ${ }^{T 2}$ : $\left.T c f 7 / 2^{f / / f l}\right)$ and $T c f 7 \mid 2 C \operatorname{trl}\left(T c f 7 / 2^{f l / f l}\right.$, or $\left.T c f 7 / 2^{f / /+}\right)$ mice were injected with tamoxifen at P9 and analyzed $24 \mathrm{~h}$ later. $B$, ISH and quantification of myelin-associated glycoprotein (Mag) mRNA. C, RT-qPCR assay for Bmp4 mRNA in the spinal cord. D, ISH showing Bmp4 mRNA upregulation in the spinal cord. $E$, ISH of Bmp4 mRNA and IHC of oligodendroglial marker Sox10 in the ventral white matter of spinal cord. $\boldsymbol{F}$, Double IHC of phosphorylated SMAD1/5 (p-SMAD) and Sox10 in the ventral white matter of spinal cord. Note that P-SMAD was barely detectable in Sox $10^{+}$cells of Tcf7I2 (trl spinal cord (arrows point to p-SMAD ${ }^{+}$Sox $10^{-}$cells) and activated in $0 C E-T c f 7 l 2$ cKO spinal cord (arrows point to $\mathrm{P}-\mathrm{SMAD}^{+}$Sox $10^{+}$cells). $\boldsymbol{G}, I d 1$, Id2, and Id3 expression quantified by RT-qPCR in the spinal cord. $\boldsymbol{H}-\boldsymbol{K}$, Experimental design $(\boldsymbol{H})$ and RT-qPCR quantification for $T c f 7 / 2(\boldsymbol{I}), B m p 4(\boldsymbol{J})$, and $I d 3(\boldsymbol{K})$ in the brain. Scale bars: $500 \mu \mathrm{m}(\boldsymbol{B}), 200 \mu \mathrm{m}(\boldsymbol{D}), 20 \mu \mathrm{m}(\boldsymbol{E})$, and $10 \mu \mathrm{m}(\boldsymbol{F})$. Please see Table 1 for statistical details throughout this study. 

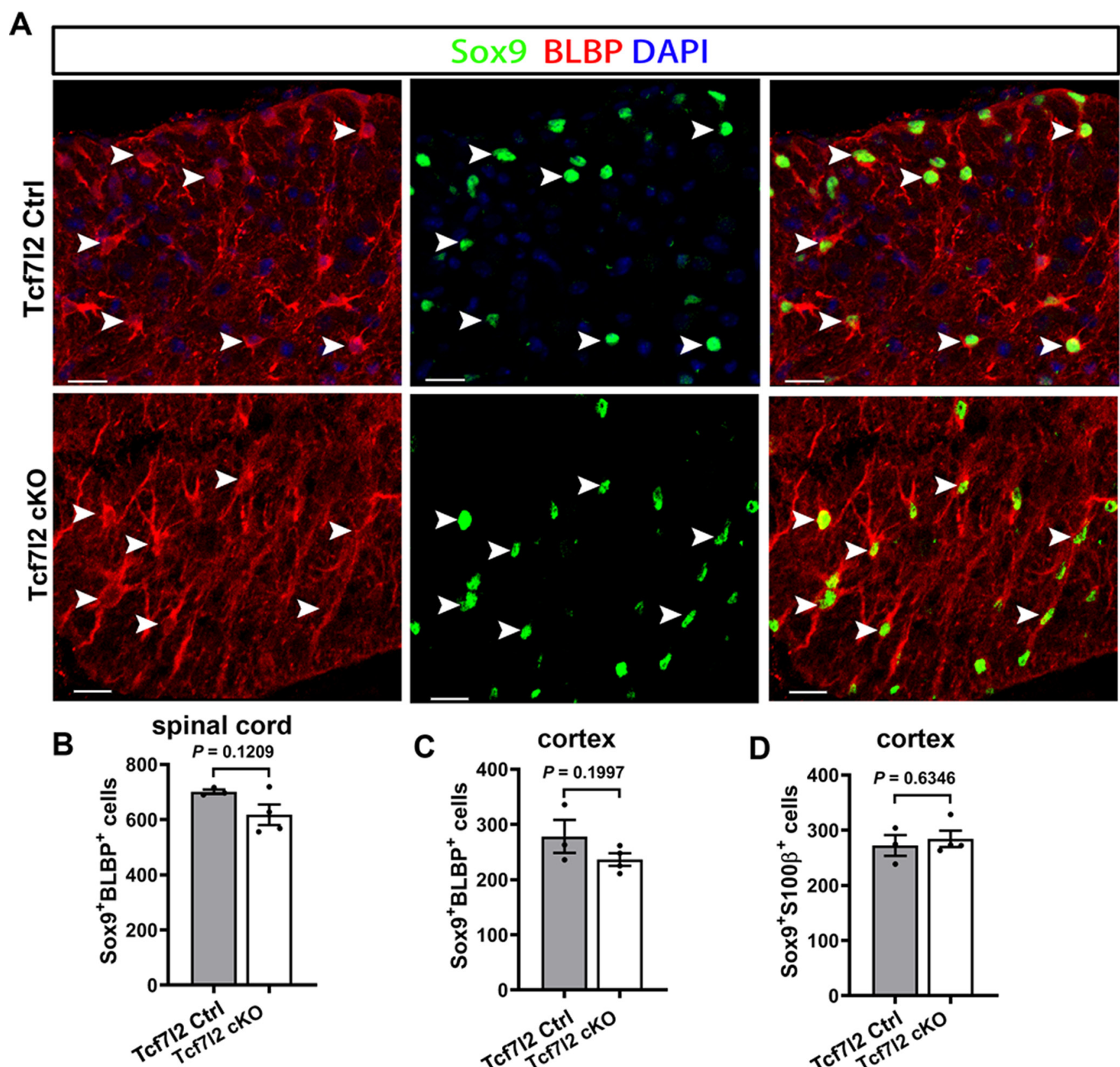

C

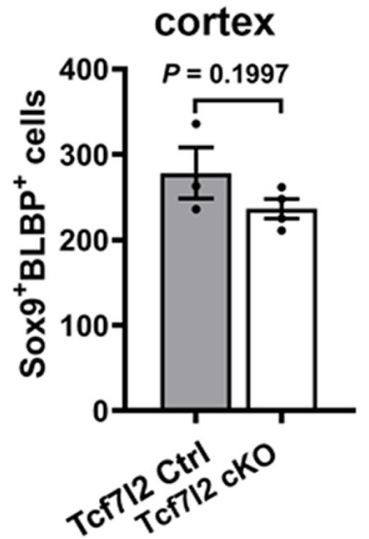

D

\section{cortex}

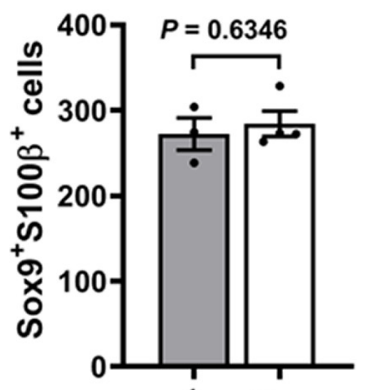

$\mathbf{E}$

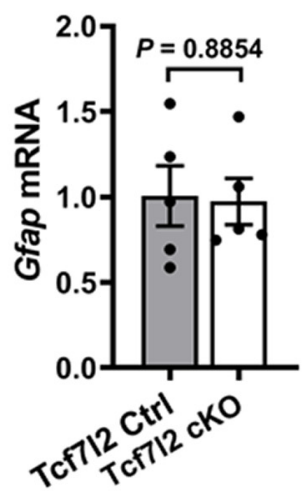

$\mathbf{F}$
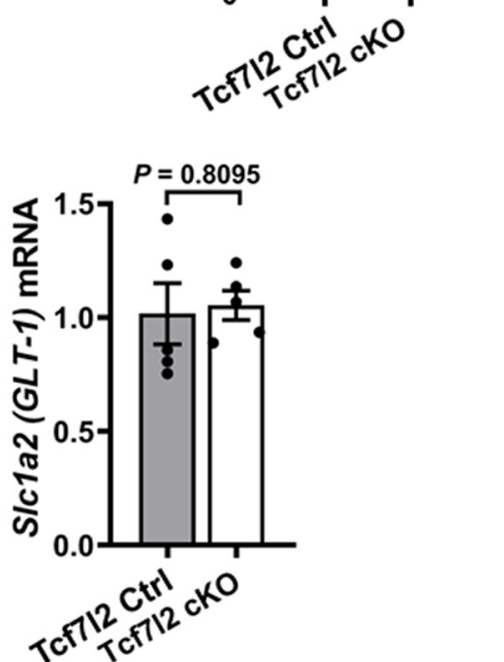

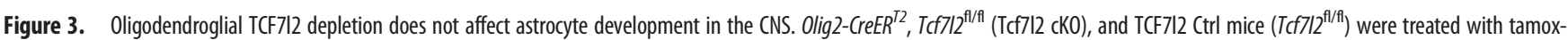
ifen at P6 and P7 and analyzed at P14. A, representative confocal images showing Sox $9^{+} \mathrm{BLBP}^{+}$astrocytes (arrowheads) in the spinal cord white matter. Scale bar: $20 \mu \mathrm{m}$. $\boldsymbol{B}, \boldsymbol{C}$, Density $\left(\# / \mathrm{mm}^{2}\right)$ of Sox $9^{+} \mathrm{BLBP}^{+}$astrocytes in the spinal cord white matter $(\boldsymbol{B})$ and forebrain cerebral cortex (C). D, Sox $9^{+}$S $100 \beta^{+}$astrocytes in the forebrain cerebral cortex. $\boldsymbol{E}, \boldsymbol{F}, \mathrm{RT}-\mathrm{qPCR}$ quantification of mature astrocyte markers Gfap and S/c1a2 in the spinal cord. 


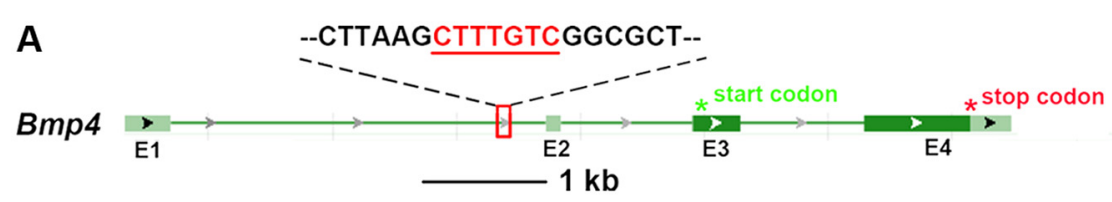

C

\begin{tabular}{l} 
mouse $T c f 7 / 2$ exon splicing \\
\begin{tabular}{|l|l|l|l|l|l|}
\hline 11 & 12 & 13 & 14 & 15 & 16 \\
\hline
\end{tabular} \\
\hline
\end{tabular}

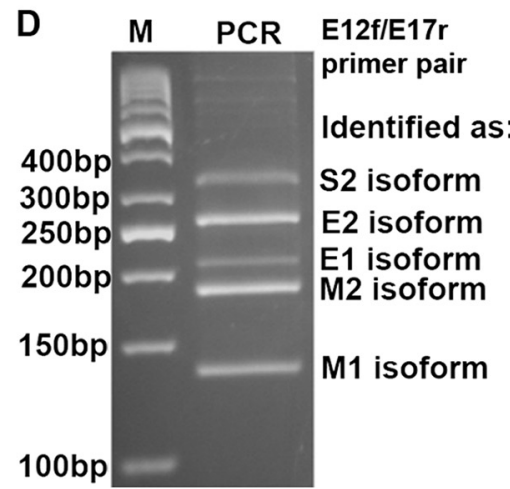

$E_{\text {Mouse spinal cord (P10) }}$

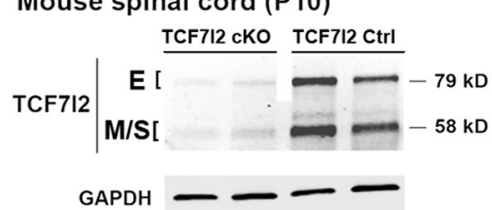

F Oli-Neu cells

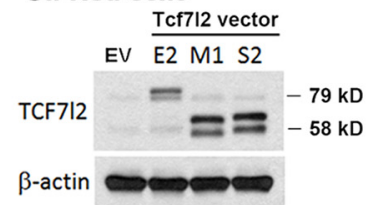

B

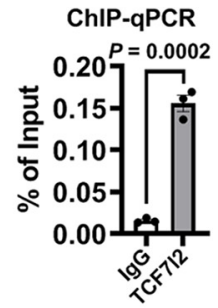

$\mathbf{G}$

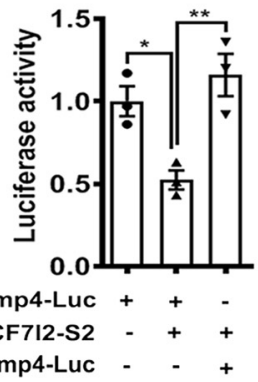

$\mathbf{H}$

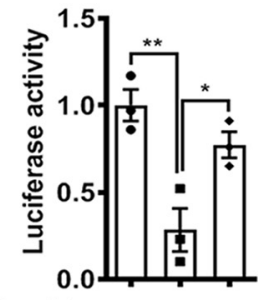

Bmp4-Luc ++ -

TCF7I2-E2 - + +

mutBmp4-Luc - - +

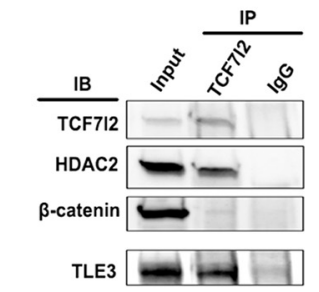

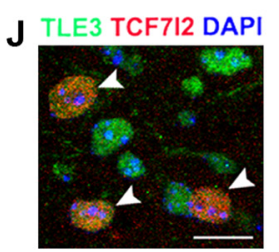

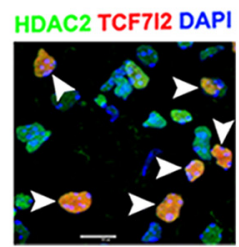

L Primary culture

TSA TSA Analysis

\begin{tabular}{l|l|l|l}
\multicolumn{1}{|c|}{$\downarrow$} & \\
\hline OPCs & D1 OLs & D2 OLs & \\
\hline
\end{tabular}

M

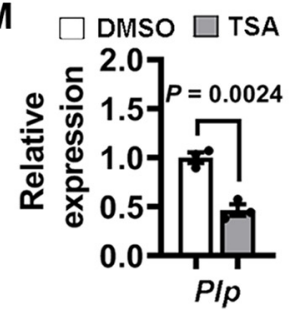

$\beta$-catenin TCF7I2 DAPI

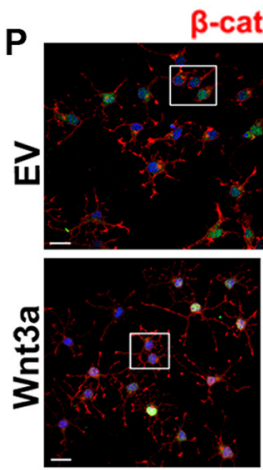

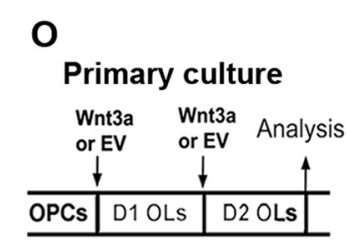

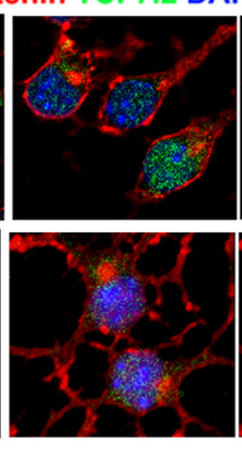

$\mathbf{N}$

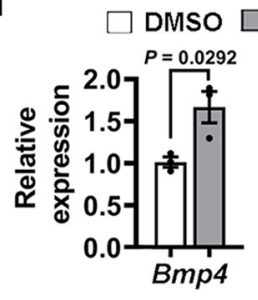

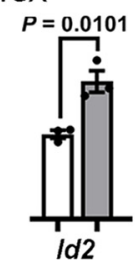
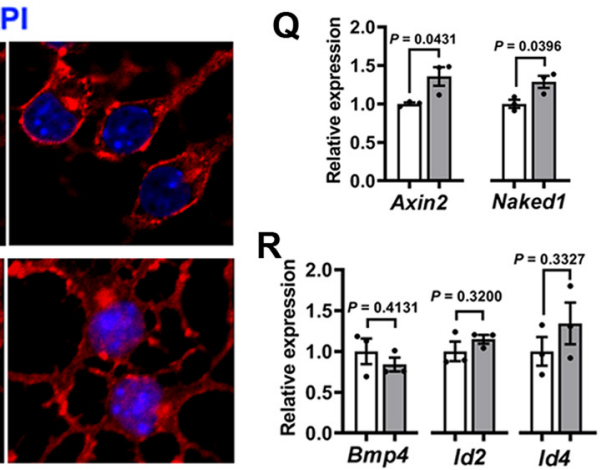

Figure 4. TCF7l2 directly represses BMP4 expression. A, Putative TCF7l2-binding site located at $\sim 317$ bp upstream of the exon 2 (E2) of the mouse Bmp4 gene. $B$, ChIP-qPCR demonstrating TCF712 binding to the intronic Bmp4 promoter shown in A. C, Schematic diagram showing alternative splicing of the mouse TCf7/2 gene (Weise et al., 2010) and PCR primer pairs (E12f and E17r) used for amplifying mRNA transcripts of different splicing isoforms. D, PCR amplicons using the CDNA prepared from P10 mouse spinal cord. The amplicons were TA-cloned and 
TCF712 have three major categories of alternative splicing isoforms (E, M, and S) depending the inclusion/exclusion of exons 13-16 (Fig. 4C; Weise et al., 2010). PCR amplification using primers targeting $T c f 7 l 2$ exon 12 and exon 17 in combination with DNA sequencing (Fig. 4C) showed that Tcf7l2 mRNA transcripts corresponding to $\mathrm{E}, \mathrm{M}$, and $\mathrm{S}$ isoforms were present in the murine spinal cord (Fig. 4D). The expression of TCF712 E isoform $(\sim 79 \mathrm{kDa})$ and $\mathrm{M} / \mathrm{S}$ isoforms $(\sim 58 \mathrm{kDa})$ was further confirmed by Western blot assay at the protein level in the spinal cord (Fig. 4E) and in primary brain OLs (Hammond et al., 2015). We therefore tested the effects of the representative E2 and S2 isoform expression (Fig. $4 F$ ) on Bmp4 transcription activity. To this end, we cloned a 439-bp intronic promoter sequence (Thompson et al., 2003) into the luciferase reporter pGL2 vector and co-transduced with TCF712-E2 or TCF712-S2 vectors into U87, a human primary glioblastoma cell line. Our results demonstrated that both E2 and S2 isoforms inhibited Bmp4luciferase activity (Fig. 4G,H). Importantly, mutation of the TCF712 binding site abolished the repressive effect (Fig. 4G, $H$ ), suggesting that TCF712 represses BMP4 expression at the transcriptional level.

TCF712 harbors several functional domains including a $\beta$-catenin-binding domain, a Groucho/TLE-binding domain, and a high-mobility group (HMG) box DNA-binding domain (Weise et al., 2010) which specifically recognizes CTTTG DNA motif on target genes (Hatzis et al., 2008). The gene regulation (activation or repression) by TCF712 is determined by its interacting co-activators or co-repressors in a cell type-dependent manner (Hurlstone and Clevers, 2002). To gain mechanistic insights into how TCF7l2 inhibits BMP4, we assessed the interaction of TCF712 with $\beta$-catenin and HDAC2 in vivo, both of which have been shown to compete for TCF712 binding (Ye et al., 2009). TCF7l2 was histologically co-labeled with HDAC2 (Fig. $4 J$ ) but not $\beta$-catenin (Fig. 4I). Co-IP followed by Western blotting demonstrated that TCF712 bound primarily to HDAC2 yet minimally to $\beta$-catenin (Fig. $4 K$ ). The binding of the co-activator $\beta$-catenin to TCF7l2 displaces the co-repressor Groucho/

\footnotetext{
sequenced. All three major isoforms $(E, S$, and $M)$ were expressed in the spinal cord. $\boldsymbol{E}$, Western blot assay for TCF7l2. Both $\sim 79-\mathrm{kDa}$ (E isoform) and $\sim 58-\mathrm{kDa}$ (M/S isoforms, similar molecular weight) bands were present in the mouse spinal cord and substantially downregulated in P10 TCF7/2 CK0 spinal cord (Olig2-CreER ${ }^{\mathrm{T} 2}$ :Tff7/ ${ }^{\mathrm{f} / \mathrm{fl}}$, tamoxifen injection at P9). TCF7I2 (clone C48H11, Cell Signaling \#2569) was used. GAPDH serves as a loading control. $\boldsymbol{F}$, Western blotting showing vector-mediated expression of the representative mouse TCF7I2 isoforms (E2, M1, and S2) in the mouse oligodendroglial cell line Oli-Neu cells. Vectors were obtained from Weise et al. (2010). EV, empty vector. $\boldsymbol{G}, \boldsymbol{H}$, Luciferase assays for Bmp4-luc and mutated Bmp4-luc (mutBmp4) in the presence of TCF7I2 S2 (G) or E2 (H) isoforms. I, J, Double IHC showing TCF7I2 is co-labeled with HDAC2 and c0-repressor TLE3 (J, arrowheads) but not $\beta$-catenin ( $\boldsymbol{I}$, dashed circles) in P7 spinal cord. Scale bar: $10 \mu \mathrm{m} . \boldsymbol{K}$, TCF7I2 Co-IP and Western blotting detection of HDAC2, $\beta$-catenin, and TLE3 in P7 spinal cord. $\boldsymbol{L}$, Experimental design for $\boldsymbol{M}, \boldsymbol{N}$. Primary OPCs were purified from neonatal mouse brain. The HDAC inhibitor TSA $(10 \mathrm{ng} / \mathrm{ml})$ or DMSO control was included in the differentiation medium for $48 \mathrm{~h}$ (medium change at $24 \mathrm{~h}$ ) before analysis. The dose was chosen based on previous study (Marin-Husstege et al., 2002). M, RT-qPCR assay showing significant reduction of mature 0L marker Plp in TSA-treated 0Ls. N, RT-qPCR assay for Bmp4 and Id2 in primary 0Ls treated with DMSO or TSA. $\mathbf{0}$, Experimental design for $\boldsymbol{P}, \boldsymbol{R}$. Primary mouse OPCs was transduced with empty control vector (EV) and vector expressing Wnt3a, a canonical Wnt ligand, at the time of differentiation and analyzed $48 \mathrm{~h}$ after transduction. $\boldsymbol{P}$, Confocal images of single optical slice showing localization of $\beta$-catenin in Wnt3a-transduced and empty vectortransduced OLs. The boxed areas were shown at higher magnification on the right. Scale bar: $10 \mu \mathrm{m} . \mathbf{Q}, \boldsymbol{R}$, Relative mRNA levels of Wnt/ $\beta$-catenin signaling targets Axin2 and Naked1 (Q) and Bmp4 and BMP4 signaling targets Id2 and Id4. White bars, Ctrl; gray bars, Wnt3a.
}

$\leftarrow$
TLE from TCF7l2 (Clevers, 2006). The co-localization and interaction of TLE3 with TCF7l2 (Fig. 4J,K) also supported a minimal $\beta$-catenin binding to TCF7l2 under physiological conditions. To determine the involvement of HDAC2 in TCF712-mediated BMP4 repression, we treated primary OLs with trichostatin A (TSA), a pan-HDAC inhibitor (Fig. $4 L$ ). In line with previous conclusion (Marin-Husstege et al., 2002; Ye et al., 2009), TSA remarkably decreased OL differentiation, evidenced by reduced expression of myelin protein gene $\mathrm{Plp}$ (Fig. $4 M$ ). Interestingly, $B m p 4$ and canonical BMP4 signaling target $I d 2$ were significantly upregulated in TSA-treated OL culture (Fig. $4 N$ ), suggesting that the deacetylation activity of HDAC2 is required for TCF7l2mediated BMP4 repression.

To determine whether "enforced" binding of $\beta$-catenin to TCF712 plays a role in TCF712-mediated BMP4 repression, we overexpressed Wnt3a, a typical canonical Wnt ligand, in primary OLs (Fig. 4O). In sharp contrast to cytoplasmic $\beta$-catenin in the control condition (Fig. 4P, upper panels), Wnt3a expression resulted in $\beta$-catenin localization in $\mathrm{TCF}_{1} 2^{+} \mathrm{DAPI}^{+}$nuclei (Fig. $4 P$, lower panels) and led to $\mathrm{Wnt} / \beta$-catenin activation, as evidenced by the elevation in canonical Wnt targets Axin2 and Naked1 (Fig. 4Q). However, Wnt3a expression did not perturb the expression of BMP4 and canonical BMP/SMAD signaling targets $I d 2$ and $I d 4$ (Fig. $4 R$ ). Together, these data suggest that TCF7l2 inhibits BMP4 expression through recruiting the repressive co-factors HDAC2 and TLE3 to Bmp4 cis-regulatory element and that $\beta$-catenin plays a dispensable role in TCF712mediated BMP4 repression.

\section{TCF712 promotes OL differentiation by repressing BMP4 in vitro}

To determine the biological significance of TCF712 in repressing BMP4, we overexpressed TCF712 in primary OLs and analyzed OL differentiation (Fig. 5A-C). TCF712 overexpression remarkably elevated the density of ramified $\mathrm{MBP}^{+}$Sox $10^{+}$ OLs (Fig. $5 D$, left) by a greater than 2 -fold (\% ramified Sox $10^{+} \mathrm{MBP}^{+}$cells: $8.34 \pm 0.45 \% \mathrm{EV}, 18.97 \pm 2.80 \%$ TCF712OE, $t_{(4)}=3.75, p=0.0199$; Fig. $5 D$, right) and significantly increased the expression of myelin protein genes $(M b p, P l p$, and $M o g$ ), newly differentiated OL marker Enpp6, and prodifferentiation factor Myrf (Fig. 5E), indicating that TCF712 is sufficient for promoting OL differentiation in a cell autonomous manner. Consistent with the inhibitory role in BMP4 transcription, TCF712 overexpression reduced the amount of BMP4 protein secreted into the culture medium (Fig. 5F), decreased the number of Sox $10^{+}$OLs that were positive for $\mathrm{p}$ SMAD1/5 (\% p-SMAD ${ }^{+}$Sox $10^{+}$cells: $36.5 \pm 1.1 \%$ EV, $28.9 \pm$ $2.0 \%$ TCF712-OE, $t_{(4)}=3.298, p=0.03$; Fig. $5 G$ ), and reduced the expression of the canonical BMP4 signaling target Id2 (Fig. $5 \mathrm{H}$ ), suggesting that TCF7l2 promotes OL differentiation by inhibiting autocrine BMP4 and dampens BMP4-mediated canonical BMP/SMAD signaling activity. We did not find significant differences in the expression of canonical Wnt target genes (Guo et al., 2015) in between EV and TCF712-OE groups (Fig. 5I), indicating that TCF7l2 plays a minor role in transcriptionally activating $\mathrm{Wnt} / \beta$-catenin signaling in oligodendroglial lineage cells (Hammond et al., 2015). BMP4 treatment (Fig. 5J) elevated the canonical BMP/SMAD signaling activity in primary OLs as evidenced by Id2 upregulation on BMP4 treatment (Fig. $5 K)$. We found that BMP4 treatment abolished the upregulation of Plp expression elicited by TCF7l2 overexpression (Fig. 5L). Collectively, these data suggest that TCF7l2 promotes OL 
A Primary culture

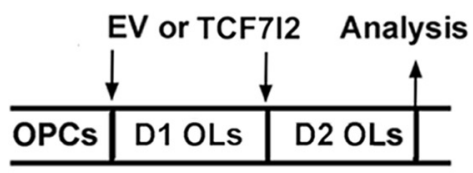

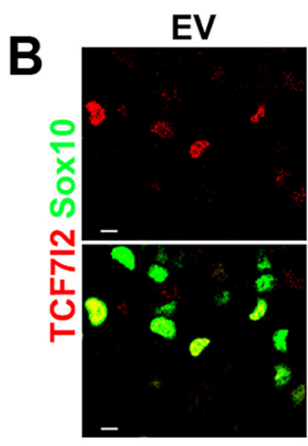

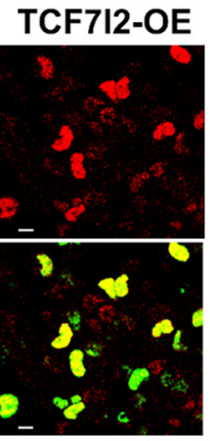

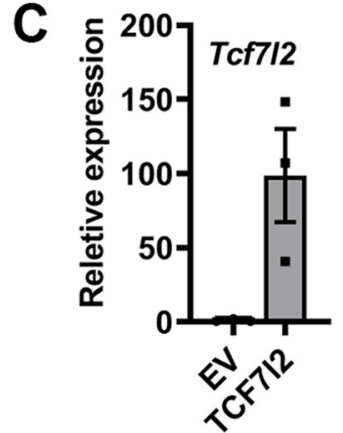
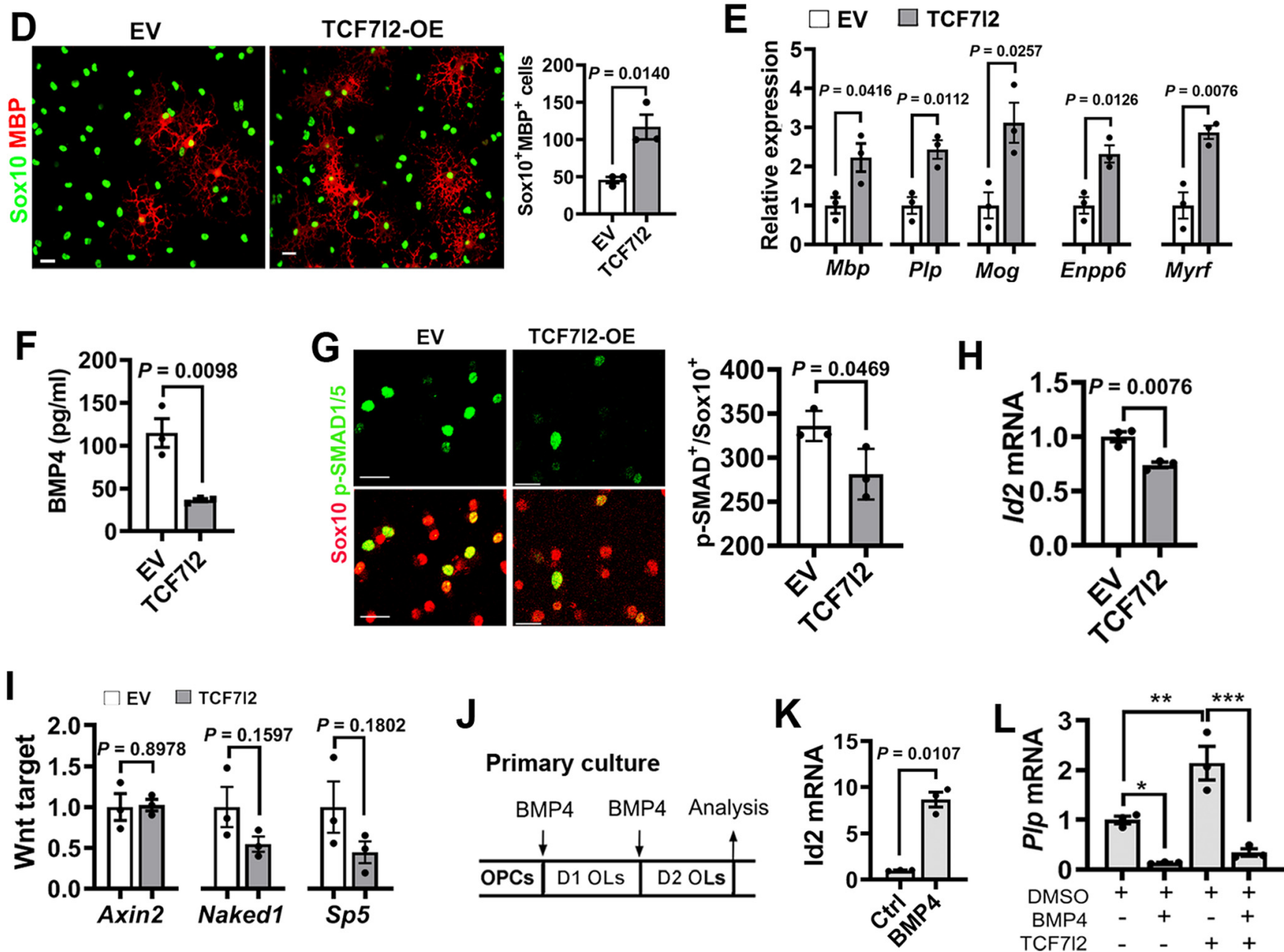

Figure 5. TCF7I2 overexpression represses BMP4-mediated signaling and promotes $0 \mathrm{~L}$ differentiation in vitro. $\boldsymbol{A}$, Experimental design for B-I. Primary $0 \mathrm{Ls}$ were transduced with empty vector (EV) or S2 isoform of TCF712 plasmid (TCF712 overexpression, TCF712-OE), and analyzed at $48 \mathrm{~h}$ after transduction. $\boldsymbol{B}, \boldsymbol{C}$, Representative confocal images of TCF712 and Sox10 (B) and RT-qPCR assay of $T C f 7 / 2$ mRNA $(C)$ in EV and TCF7/2-0E groups. $\boldsymbol{D}$, ICC and quantification of Sox $10^{+} \mathrm{MBP}^{+}$cells transduced with EV or TCF7|2-0E vector. $E$, RT-qPCR quantification for myelin genes, newly formed OL marker Enpp6, and prodifferentiation factor Myrf. $\boldsymbol{F}$, ELISA assay for BMP4 concentration in culture medium. $\boldsymbol{G}$, Representative confocal images and density of p-SMAD1/ $5^{+}$Sox $10^{+}$cells $\left(\# / \mathrm{mm}^{2}\right)$. $\boldsymbol{H}$, relative mRNA levels of BMP4 signaling target Id2 quantified by RT-qPCR. I, Relative expression of canonical Wnt targets quantified by RT-qPCR. J, Experimental design for $\boldsymbol{K}, \boldsymbol{L}$. BMP4 $(10 \mathrm{ng} / \mathrm{ml})$ or DMSO control was included in the differentiation medium for $48 \mathrm{~h}$ (medium change at $24 \mathrm{~h}$ ) before analysis. $\boldsymbol{K}$, RT-qPCR quantification of canonical BMP4 signaling target Id2. Two-tailed $t$ test, Welch's corrected $t_{(2.009)}=9.529$. L, RT-qPCR assay for OL marker Plp in primary OLs treated with BMP4, TCF7l2-0E, or both. Scale bars: $10 \mu \mathrm{m}(\boldsymbol{B}, \mathbf{D}, \mathbf{G})$.

differentiation through controlling autocrine BMP4-mediated signaling.

Disrupting oligodendroglial BMP4 rescues OL differentiation defects in TCF712 cKO mutants To determine the physiological significance of TCF712-mediated BMP4 repression on OL differentiation in vivo, we generated Olig2-CreER ${ }^{\mathrm{T} 2}, T c f 7 l 2^{\mathrm{fl} / \mathrm{fl}}$ (TCF7l2 single cKO), Olig2-CreER ${ }^{\mathrm{T} 2}$,
$T c f 7 l 2^{\mathrm{fl} / \mathrm{fl}}, B m p 4^{\mathrm{fl} / \mathrm{fl}}(\mathrm{TCF} 712 / \mathrm{BMP} 4$ double $\mathrm{cKO})$, and control mice. In line with the above observation (Fig. 2), TCF7l2 disruption (Fig. 6A,B) caused BMP4 upregulation (Fig. 6C) and BMP4/ SMAD signaling activation (Fig. $6 D, E$ ) in Sox $10^{+}$oligodendroglial lineage cells (Fig. $6 F, G$ ) of TCF712 single cKO mutants. Simultaneous Bmp4 deletion (Fig. $6 \mathrm{C}$ ) normalized BMP/SMAD signaling activity in TCF7l2/BMP4 double cKO mice to the level in control mice (Fig. 6D-G). 

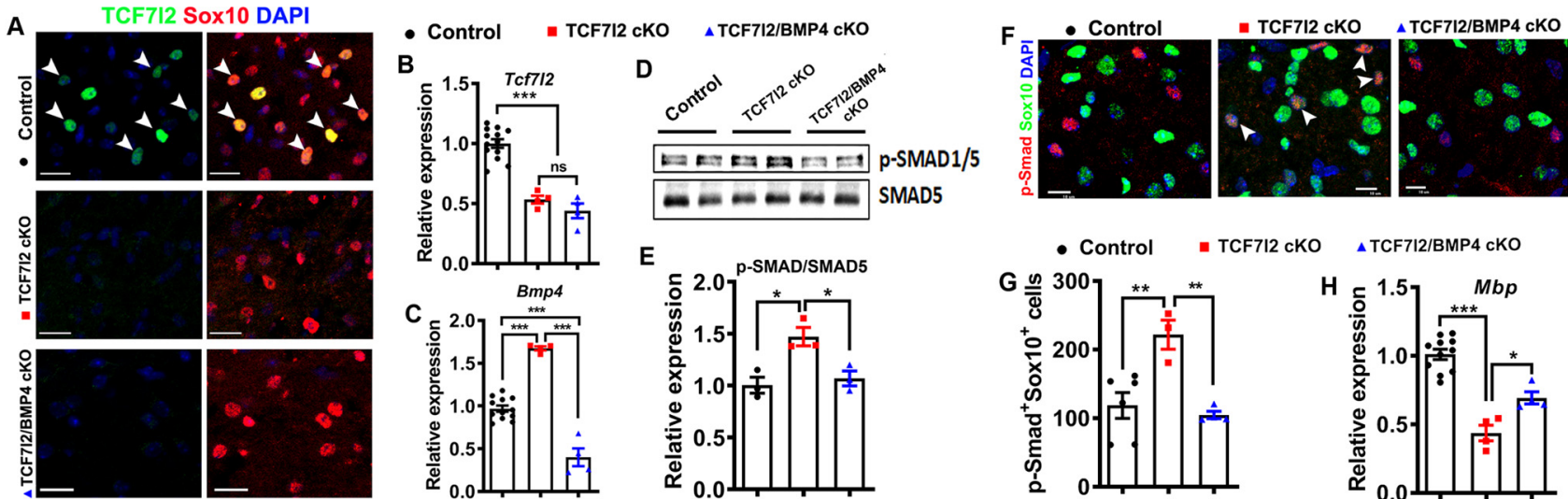

- Control
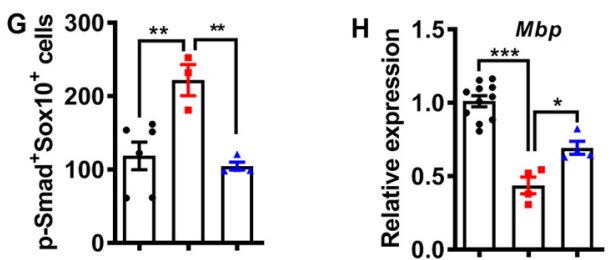
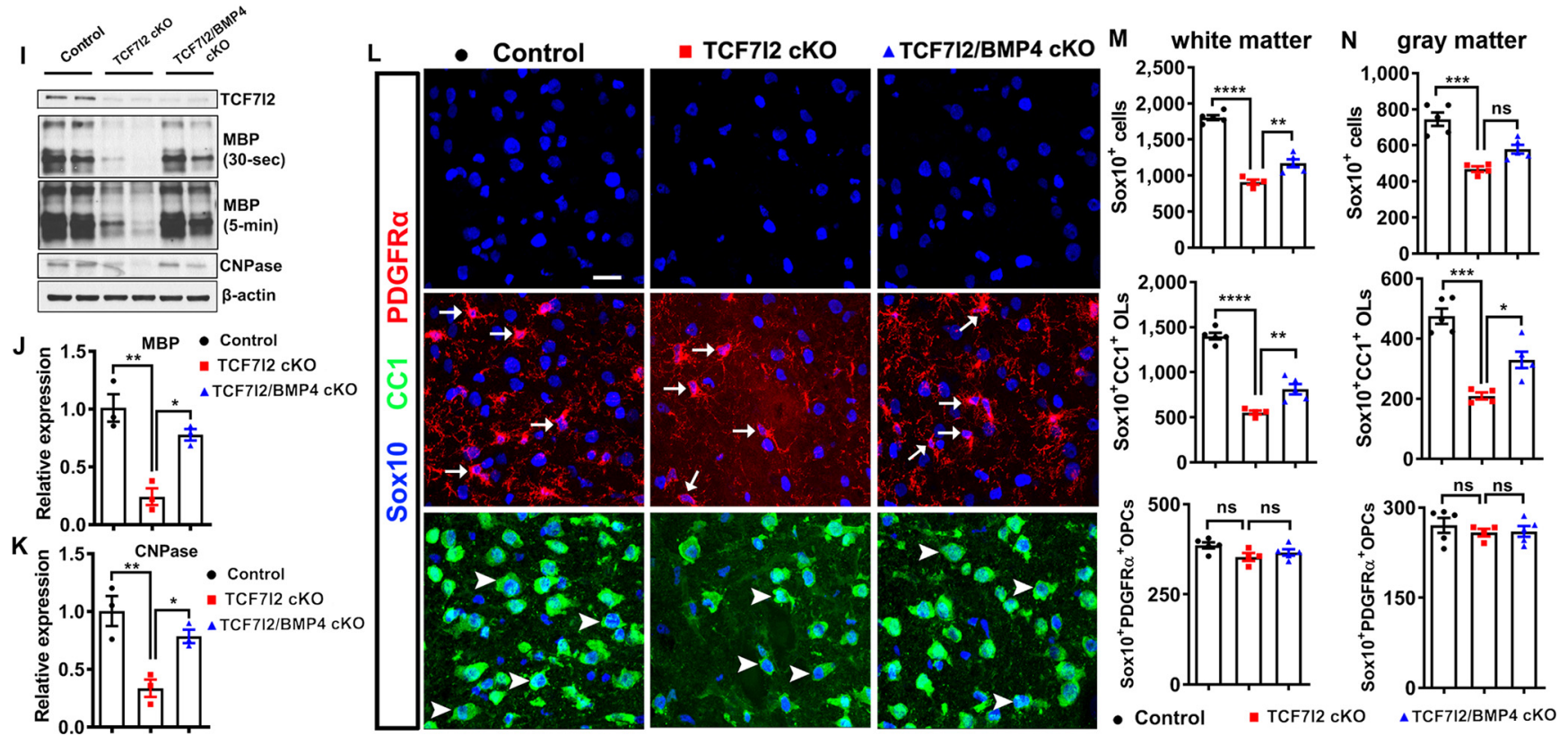

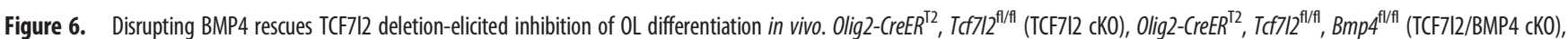
and control mice were treated with tamoxifen at P6 and P7 and analyzed at P13. $\boldsymbol{A}$, Representative confocal images of TCF712 and Sox10 co-labeling cells (arrowheads) in the spinal cord. $\boldsymbol{B}, \boldsymbol{C}$, Relative expression of $T \subset f 7 / 2$ and $B m p 4$ in the spinal cord assessed by RT-qPCR. $\boldsymbol{D}, \boldsymbol{E}$, Representative Western blot images and quantification of p-SMAD1/5 over SMAD5 in the forebrain. $\boldsymbol{F}, \boldsymbol{G}$, Representative confocal images and quantification of p-SMAD1/5 and Sox10 IHC $\left(\# / \mathrm{mm}^{2}\right)$ in the spinal cord white matter. $\boldsymbol{H}$, Relative Mbp expression quantified by RT-qPCR. I- $\boldsymbol{K}$, Representative Western blot images and quantification of TCF7I2, MBP, and CNPase in the forebrain. $\beta$-Actin serves as an internal loading control. $L$, Representative confocal images of Sox10, $\mathrm{CC} 1$, and PDGFR $\alpha \mathrm{IHC}$ in the spinal cord white matter. Arrowheads, Sox $10^{+} \mathrm{CC} 1^{+}$OLs; arrows, Sox $10^{+} \mathrm{PDGFR} \alpha^{+}$OPCs. $\boldsymbol{M}, \mathbf{N}$, Densities (\#/mm ${ }^{2}$ ) of total Sox $10^{+}$oligodendroglial lineage cells, Sox $10^{+} \mathrm{CC}^{+}$OLs, and Sox $10^{+}$PDGFR $\alpha^{+}$OPCs in the white matter $(\boldsymbol{M})$ and gray matter $(\boldsymbol{N})$ of spinal cord. Scale bars: $20 \mu \mathrm{m}(\boldsymbol{A})$ and $10 \mu \mathrm{m}(\boldsymbol{F}, \boldsymbol{L})$.

Consistent with previous reports (Hammond et al., 2015; Zhao et al., 2016), TCF712 deficiency remarkably decreased the expression of myelin basic protein MBP and CNPase at the mRNA (Fig. 6H) and protein level (Fig. 6I-K) and significantly diminished OL differentiation (Fig. $6 L-N$ ) in TCF712 single cKO mice compared with control mice. Simultaneous BMP4 deletion remarkably alleviated the defects in myelin gene expression in TCF712/BMP4 double cKO compared with TCF712 single cKO mice (Fig. $6 \mathrm{H}-\mathrm{K}$ ). Since the number of oligodendroglial lineage cells in the white matter is much greater than that in the gray matter, we quantified Sox $10^{+}$cells, Sox $10^{+} \mathrm{CC}^{+}$ differentiated OLs, and Sox $10^{+}$PDGFR $\alpha^{+}$OPCs (Fig. $6 \mathrm{~L}$ ) in the white matter and gray matter separately. Our data demonstrated that disrupting BMP4 significantly increased the number of Sox $10^{+} \mathrm{CCl}^{+}$differentiated OLs in the white matter (Fig. 6M) and gray matter (Fig. 6N) of TCF712/BMP4 double cKO compared with that in TCF712 single cKO mice. Thus, our genetic results convincingly demonstrate that
BMP4 dysregulation is a downstream molecular mechanism underlying OL differentiation defects elicited by TCF712 cKO in vivo.

To determine the effect of BMP4 depletion alone on OL differentiation in vivo, we analyzed Pdgfr $\alpha-C r e E R^{\mathrm{T} 2}, B m p 4^{\mathrm{Al} / \mathrm{fl}}$ (BMP4 cKO) mice and age-matched control mice. Despite a greater than 4 -fold BMP4 reduction in BMP4 $\mathrm{cKO}$ mice compared with BMP4 Ctrl littermates (Fig. 7A), we did not find significant differences in the number of differentiated OLs and OPCs (Fig. $7 B-D$ ), myelin gene expression (Fig. $7 E$ ), or the rate of OL differentiation indicated by the number of $\mathrm{APC}^{+} \mathrm{TCF}^{+} 2^{+}$ (Zhang et al., 2018a) newly generated OLs (Fig. $7 F-I$ ). Despite a $>4$-fold reduction in BMP4, BMP4 cKO mice maintained a comparatively normal level of BMP/SMAD signaling activity, as assessed by similar levels of canonical BMP/SMAD target genes Id1 and Id2 between BMP4 Ctrl and cKO mice (Fig. 7J). These data suggest that other BMP ligands, for example, BMP2 and BMP6 from other cell types (See et al., 2007), may be redundant 

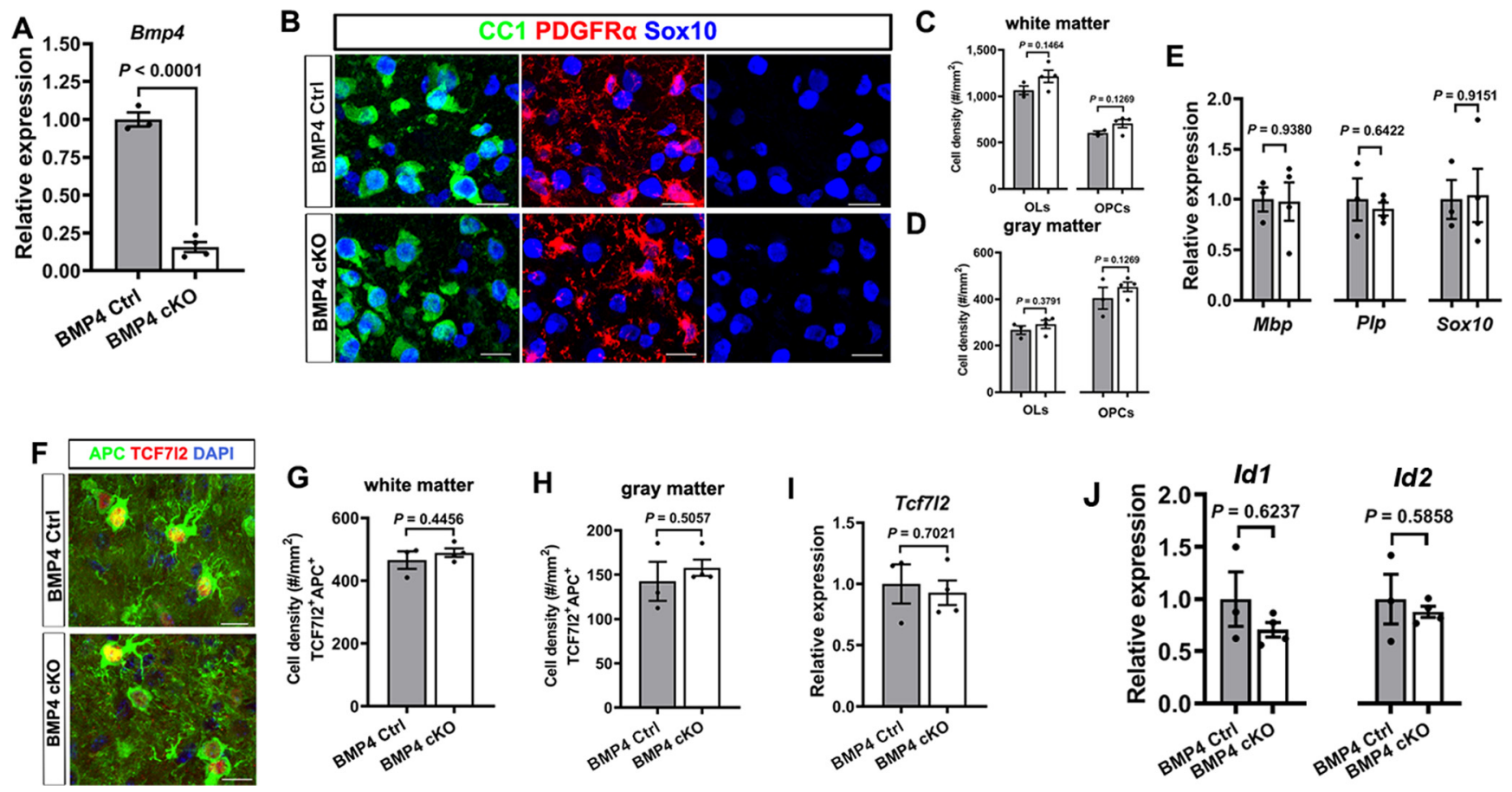

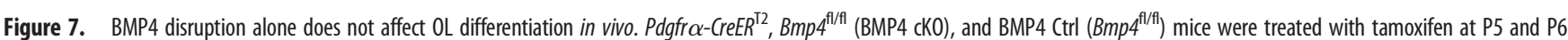
and the spinal cord was analyzed at P10. $\boldsymbol{A}$, Relative Bmp4 expression by RT-qPCR. B-D, Representative images of Sox10/CC1/PDGFR $\alpha$ IHC $(\boldsymbol{B})$ and densities of Sox $10^{+} \mathrm{CC} 1^{+}$0Ls and Sox $10^{+}$PDGFR $\alpha^{+}$OPCs in the spinal cord white $(\boldsymbol{C})$ and gray (D) matter. Scale bar: $10 \mu \mathrm{m}$. $\boldsymbol{E}$, Relative expression of myelin protein gene Mbp and Plp and pan-oligodendroglial marker Sox 10 quantified by RT-qPCR. $\boldsymbol{F}-\boldsymbol{H}$, Representative confocal image of newly differentiated 0Ls labeled by APC/TCF7I2 $(\boldsymbol{F})$, and quantification of APC ${ }^{+} / \mathrm{TCF} 72^{+}$cells in the white $(\boldsymbol{G})$ and gray $(\boldsymbol{H})$ matter. Scale bar: $10 \mu \mathrm{m}$. I, Relative Tcf7/2 expression in the spinal cord by RT-qPCR. J, Relative expression of canonical BMP signaling targets Id1 and Id2 quantified by RT-qPCR.

with BMP4 in maintaining normal BMP/SMAD signaling activity and OL differentiation in BMP4 cKO mice.

\section{Discussion}

By employing in vitro primary culture and genetic mouse models in combination with biochemical and molecular analyses, we made several novel observations: (1) oligodendroglial lineage is the primary cellular source of BMP4 in the CNS; (2) TCF712 controls the level of autocrine BMP4 signaling activity in oligodendroglial lineage cells by transcriptionally repressing Bmp4 gene; and (3) TCF7l2 promotes OL differentiation by dampening autocrine BMP4mediatd signaling in vitro and in vivo.

Our previous studies have shown that TCF712 is transiently upregulated in newly formed OLs (Hammond et al., 2015) that are positive for APC, a Wnt $\beta$-catenin negative regulator (Lang et al., 2013; Fig. 8A). Based on our findings, we proposed a "Wnt-independent and BMP4-dependent" role of TCF712 in promoting OL differentiation (Fig. $8 B$ ). In this working model, APC limits the nuclear availability of $\beta$-catenin for TCF7l2 binding and $\mathrm{Wnt} / \beta$-catenin activation whereas TCF712 binds to the cis-regulatory elements of Bmp4 genes and directly represses Bmp4 transcription likely by recruiting co-repressors HDAC2 and TLE3, both of which lack DNA-binding domains. Disrupting the DNA-binding TCF712 leads to uncontrolled BMP4 expression and inhibits OL differentiation through BMP4-mediated autocrine signaling activation. The interaction of TCF7l2 with HDAC2 and the effect of the HDAC inhibitor TSA on increasing BMP4 are in line with previous data showing that histone deacetylation is required for OPCs to differentiate into mature OLs (Shen et al., 2005).

Previous data show that $\mathrm{Wnt} / \beta$-catenin signaling regulates the timely differentiation of OPCs into OLs (Dai et al., 2014). Our working model of Wnt-independent function of TCF712 does not conflict with the conclusion by Qiu and colleagues (Dai et al., 2014). The TCF/LEF family consists of TCF7, TCF711, TCF712, and LEF1 all of which could transcriptionally mediate $\mathrm{Wnt} / \beta$-catenin signaling. It is possible that other TCF/LEF family member may mediate the effect of $\mathrm{Wnt} / \beta$-catenin signaling on oligodendroglial differentiation. Consistent with this possibility, our previous data (Hammond et al., 2015) demonstrate that $\mathrm{Wnt} / \beta$-catenin signaling activation elicited by APC disruption (Lang et al., 2013) remarkably upregulates the expression of the Wnt effector LEF1 in oligodendroglial lineage cells. We are current employing oligodendroglial-specific APC/LEF1 double cKO system to prove or disprove our hypothesis. Our in vivo rescue experiments demonstrated that ablating oligodendroglial BMP4 partially rescued the inhibitory degree of OL 
differentiation elicited by TCF712 depletion (Fig. 6M,N), suggesting that other molecular mechanisms may also play a part in TCF712-regualated OL differentiation.

TCF712 is upregulated primarily in active MS lesions (with extensive remyelination) but minimally in chronic MS lesions (with scarce remyelination; Fancy et al., 2009). Recent studies demonstrate an elevation of BMP4 in both active and chronic MS lesions (Costa et al., 2019; Harnisch et al., 2019) although the cell types producing BMP4 need to be defined (Grinspan, 2020). The repressive TCF712-BMP4 regulation suggests that augmenting TCF712 may have synergistic potential in dampening BMP4 signaling, which is dysregulated in chronic MS lesions (Grinspan, 2015, 2020) and in promoting OL differentiation and remyelination given the multimodal actions of TCF712 in propelling OL lineage progression and maturation (Zhao et al., 2016).

The proposed working model (Fig. $8 \mathrm{~B}$ ) may also be applicable to a broader cell lineage. Beyond its role in CNS oligodendroglial lineage, the Wnt effector TCF712 regulates a spectrum of biological processes of other lineage cells, such as hepatic metabolism (Boj et al., 2012), colorectal tumor genesis (Angus-Hill et al., 2011; Wenzel et al., 2020), adipocyte function (Chen et al., 2018), and heart development (Ye et al., 2019), where BMP signaling plays crucial roles (Wang et al., 2014; Brazil et al., 2015). The connection between TCF712 and autocrine BMP4 signaling has not been reported in these cell types. In this regard, our takehome message (Fig. $8 B$ ) point to a new direction for studying the crosstalk between TCF712 and BMP4/SMAD signaling pathways in regulating the development and function of TCF712-expressing and BMP/Wnt-operative cells in the body. Supporting this claim, recent data suggest that TCF7l2 overexpression downregulates Bmp4 mRNA in a cardiac cell line (Ye et al., 2019).

In summary, our in vitro and in vivo data unravel a previously unrecognized role of the Wnt effector TCF712 in promoting OL differentiation by negatively controlling BMP4-mediated autocrine signaling. Given that BMP4 is dysregulated in chronic MS lesions, our findings of the biological function of TCF712-BMP4 connection in OL differentiation indicate that enforced TCF712 expression may be a potential therapeutic option in promoting $\mathrm{OL}$ and myelin regeneration in chronic MS lesions and animal models.

\section{References}

Angus-Hill ML, Elbert KM, Hidalgo J, Capecchi MR (2011) T-cell factor 4 functions as a tumor suppressor whose disruption modulates colon cell proliferation and tumorigenesis. Proc Natl Acad Sci USA 108:4914-4919.

Armstrong RC, Dorn HH, Kufta CV, Friedman E, Dubois-Dalcq ME (1992) Pre-oligodendrocytes from adult human CNS. J Neurosci 12:1538-1547.

Boj SF, van Es JH, Huch M, Li VSW, José A, Hatzis P, Mokry M, Haegebarth A, van den Born M, Chambon P, Voshol P, Dor Y, Cuppen E, Fillat C, Clevers H (2012) Diabetes risk gene and Wnt effector Tcf712/TCF4 controls hepatic response to perinatal and adult metabolic demand. Cell 151:1595-1607.

Brazil DP, Church RH, Surae S, Godson C, Martin F (2015) BMP signalling: agony and antagony in the family. Trends Cell Biol 25:249-264.

Cadigan KM (2012) TCFs and Wnt/beta-catenin signaling: more than one way to throw the switch. Curr Top Dev Biol 98:1-34.

Chen X, Ayala I, Shannon C, Fourcaudot M, Acharya NK, Jenkinson CP, Heikkinen S, Norton L (2018) The diabetes gene and Wnt pathway effector TCF7L2 regulates adipocyte development and function. Diabetes 67:554-568.

Clevers H (2006) Wnt/beta-catenin signaling in development and disease. Cell 127:469-480.

Costa C, Eixarch H, Martínez-Sáez E, Calvo-Barreiro L, Calucho M, Castro Z, Ortega-Aznar A, Ramón Y Cajal S, Montalban X, Espejo C (2019)
Expression of bone morphogenetic proteins in multiple sclerosis lesions. Am J Pathol 189:665-676.

Dai ZM, Sun S, Wang C, Huang H, Hu X, Zhang Z, Lu QR, Qiu M (2014) Stage-specific regulation of oligodendrocyte development by Wnt/ $\beta$-catenin signaling. J Neurosci 34:8467-8473.

Fancy SP, Baranzini SE, Zhao C, Yuk DI, Irvine KA, Kaing S, Sanai N, Franklin RJ, Rowitch DH (2009) Dysregulation of the Wnt pathway inhibits timely myelination and remyelination in the mammalian CNS. Genes Dev 23:1571-1585.

Franklin RJ, Gallo V (2014) The translational biology of remyelination: past, present, and future. Glia 62:1905-1915.

Fu H, Cai J, Clevers H, Fast E, Gray S, Greenberg R, Jain MK, Ma Q, Qiu M, Rowitch DH, Taylor CM, Stiles CD (2009) A genome-wide screen for spatially restricted expression patterns identifies transcription factors that regulate glial development. J Neurosci 29:11399-11408.

Gomes WA, Mehler MF, Kessler JA (2003) Transgenic overexpression of BMP4 increases astroglial and decreases oligodendroglial lineage commitment. Dev Biol 255:164-177.

Grinspan JB (2015) Bone morphogenetic proteins: inhibitors of myelination in development and disease. Vitam Horm 99:195-222.

Grinspan JB (2020) Inhibitors of myelination and remyelination, bone morphogenetic proteins, are upregulated in human neurological disease. Neurochem Res 45:656-662.

Guo F, Maeda Y, Ko EM, Delgado M, Horiuchi M, Soulika A, Miers L, Burns T, Itoh T, Shen H, Lee E, Sohn J, Pleasure D (2012) Disruption of NMDA receptors in oligodendroglial lineage cells does not alter their susceptibility to experimental autoimmune encephalomyelitis or their normal development. J Neurosci 32:639-645.

Guo F, Lang J, Sohn J, Hammond E, Chang M, Pleasure D (2015) Canonical Wnt signaling in the oligodendroglial lineage-puzzles remain. Glia 63:1671-1693.

Hammond E, Lang J, Maeda Y, Pleasure D, Angus-Hill M, Xu J, Horiuchi M, Deng W, Guo F (2015) The Wnt effector transcription factor 7-like 2 positively regulates oligodendrocyte differentiation in a manner independent of Wnt $/ \beta$-catenin signaling. J Neurosci 35:5007-5022.

Harlow DE, Honce JM, Miravalle AA (2015) Remyelination therapy in multiple sclerosis. Front Neurol 6:257.

Harnisch K, Teuber-Hanselmann S, Macha N, Mairinger F, Fritsche L, Soub D, Meinl E, Junker A (2019) Myelination in multiple sclerosis lesions is associated with regulation of bone morphogenetic protein 4 and its antagonist noggin. Int J Mol Sci 20:154.

Hatzis P, van der Flier LG, van Driel MA, Guryev V, Nielsen F, Denissov S, Nijman IJ, Koster J, Santo EE, Welboren W, Versteeg R, Cuppen E, van de Wetering M, Clevers H, Stunnenberg HG (2008) Genome-wide pattern of TCF7L2/TCF4 chromatin occupancy in colorectal cancer cells. Mol Cell Biol 28:2732-2744.

He Y, Dupree J, Wang J, Sandoval J, Li J, Liu H, Shi Y, Nave KA, CasacciaBonnefil P (2007) The transcription factor Yin Yang 1 is essential for oligodendrocyte progenitor differentiation. Neuron 55:217-230.

Hull V, Wang Y, Burns T, Zhang S, Sternbach S, McDonough J, Guo F, Pleasure D (2020) Antisense oligonucleotide reverses leukodystrophy in Canavan disease mice. Ann Neurol 87:480-485.

Hurlstone A, Clevers H (2002) T-cell factors: turn-ons and turn-offs. EMBO J 21:2303-2311.

Lang J, Maeda Y, Bannerman P, Xu J, Horiuchi M, Pleasure D, Guo F (2013) Adenomatous polyposis coli regulates oligodendroglial development. J Neurosci 33:3113-3130.

Marin-Husstege M, Muggironi M, Liu A, Casaccia-Bonnefil P (2002) Histone deacetylase activity is necessary for oligodendrocyte lineage progression. J Neurosci 22:10333-10345.

Reiprich S, Cantone M, Weider M, Baroti T, Wittstatt J, Schmitt C, Küspert M, Vera J, Wegner M (2017) Transcription factor Sox10 regulates oligodendroglial Sox9 levels via microRNAs. Glia 65:1089-1102.

Retting KN, Song B, Yoon BS, Lyons KM (2009) BMP canonical Smad signaling through Smad1 and Smad5 is required for endochondral bone formation. Development 136:1093-1104.

See J, Mamontov P, Ahn K, Wine-Lee L, Crenshaw EB 3rd, Grinspan JB (2007) BMP signaling mutant mice exhibit glial cell maturation defects. Mol Cell Neurosci 35:171-182.

Shen S, Li J, Casaccia-Bonnefil P (2005) Histone modifications affect timing of oligodendrocyte progenitor differentiation in the developing rat brain. J Cell Biol 169:577-589. 
Takebayashi H, Nabeshima Y, Yoshida S, Chisaka O, Ikenaka K, Nabeshima Y (2002) The basic helix-loop-helix factor olig2 is essential for the development of motoneuron and oligodendrocyte lineages. Curr Biol $12: 1157-1163$

Thompson DL, Gerlach-Bank LM, Barald KF, Koenig RJ (2003) Retinoic acid repression of bone morphogenetic protein 4 in inner ear development. Mol Cell Biol 23:2277-2286.

Wang RN, Green J, Wang Z, Deng Y, Qiao M, Peabody M, Zhang Q, Ye J, Yan Z, Denduluri S, Idowu O, Li M, Shen C, Hu A, Haydon RC, Kang R, Mok J, Lee MJ, Luu HL, Shi LL (2014) Bone morphogenetic protein (BMP) signaling in development and human diseases. Genes Dis 1:87105.

Wang Y, Liu Q, Xu Y, Zhang Y, Lv Y, Tan Y, Jiang N, Cao G, Ma X, Wang J, Cao Z, Yu B, Kou J (2016) Ginsenoside Rg1 protects against oxidative stress-induced neuronal apoptosis through myosin IIA-actin related cytoskeletal reorganization. Int J Biol Sci 12:1341-1356.

Wang Y, Xu Y, Liu Q, Zhang Y, Gao Z, Yin M, Jiang N, Cao G, Yu B, Cao Z, Kou J (2017) Myosin IIA-related actomyosin contractility mediates oxidative stress-induced neuronal apoptosis. Front Mol Neurosci 10:75.

Weise A, Bruser K, Elfert S, Wallmen B, Wittel Y, Wöhrle S, Hecht A (2010) Alternative splicing of Tcf7l2 transcripts generates protein variants with differential promoter-binding and transcriptional activation properties at Wnt/beta-catenin targets. Nucleic Acids Res 38:1964-1981.

Wenzel J, Rose K, Haghighi EB, Lamprecht C, Rauen G, Freihen V, Kesselring R, Boerries M, Hecht A (2020) Loss of the nuclear Wnt pathway effector TCF7L2 promotes migration and invasion of human colorectal cancer cells. Oncogene 39:3893-3909.
Wu M, Hernandez M, Shen S, Sabo JK, Kelkar D, Wang J, O’Leary R, Phillips GR, Cate HS, Casaccia P (2012) Differential modulation of the oligodendrocyte transcriptome by sonic hedgehog and bone morphogenetic protein 4 via opposing effects on histone acetylation. J Neurosci 32:66516664 .

Ye B, Li L, Xu H, Chen Y, Li F (2019) Opposing roles of TCF7/LEF1 and TCF7L2 in cyclin D2 and Bmp4 expression and cardiomyocyte cell cycle control during late heart development. Lab Invest 99:807-818.

Ye F, Chen Y, Hoang T, Montgomery RL, Zhao X-h, Bu H, Hu T, Taketo MM, van Es JH, Clevers H, Hsieh J, Bassel-Duby R, Olson EN, Lu QR (2009) HDAC1 and HDAC2 regulate oligodendrocyte differentiation by disrupting the beta-catenin-TCF interaction. Nat Neurosci 12:829-838.

Zhang S, Rasai A, Wang Y, Xu J, Bannerman P, Erol D, Tsegaye D, Wang A, Soulika A, Zhan X, Guo F (2018a) The stem cell factor Sox2 is a positive timer of oligodendrocyte development in the postnatal murine spinal cord. Mol Neurobiol 55:9001-9015.

Zhang S, Zhu X, Gui X, Croteau C, Song L, Xu J, Wang A, Bannerman P, Guo F (2018b) Sox2 is essential for oligodendroglial proliferation and differentiation during postnatal brain myelination and CNS remyelination. J Neurosci 38:1802-1820.

Zhang S, Kim B, Zhu X, Gui X, Wang Y, Lan Z, Prabhu P, Fond K, Wang A, Guo F (2020) Glial type specific regulation of CNS angiogenesis by HIFalpha-activated different signaling pathways. Nat Commun 11:2027.

Zhao C, Deng Y, Liu L, Yu K, Zhang L, Wang H, He X, Wang J, Lu C, Wu LN, Weng Q, Mao M, Li J, van Es JH, Xin M, Parry L, Goldman SA, Clevers H, et al. (2016) Dual regulatory switch through interactions of Tcf712/Tcf4 with stage-specific partners propels oligodendroglial maturation. Nat Commun 7:10883. 